\title{
Cell-free biosensors for rapid detection of water contaminants
}

\author{
Jaeyoung K. Jung $\oplus^{1,2,3,18}$, Khalid K. Alam $\oplus^{1,2,3,18}$, Matthew S. Verosloff ${ }^{2,3,4}$, Daiana A. Capdevila $\oplus^{5}$, \\ Morgane Desmau ${ }^{6}{ }^{6}$, Phillip R. Clauer ${ }^{7}$, Jeong Wook Lee ${ }^{8}$, Peter Q. Nguyen ${ }^{9}{ }^{9}$, Pablo A. Pastén $\left({ }^{10,11}\right.$, \\ Sandrine J. Matiasek ${ }^{12,13}$, Jean-François Gaillard ${ }^{6}{ }^{6}$, David P. Giedroc ${ }^{5,14}$, James J. Collins ${ }^{7,9,15,16,17}$ \\ and Julius B. Lucks $\mathbb{1}$ 1,2,3,4凶
}

Lack of access to safe drinking water is a global problem, and methods to reliably and easily detect contaminants could be transformative. We report the development of a cell-free in vitro transcription system that uses RNA Output Sensors Activated by Ligand Induction (ROSALIND) to detect contaminants in water. A combination of highly processive RNA polymerases, allosteric protein transcription factors and synthetic DNA transcription templates regulates the synthesis of a fluorescence-activating RNA aptamer. The presence of a target contaminant induces the transcription of the aptamer, and a fluorescent signal is produced. We apply ROSALIND to detect a range of water contaminants, including antibiotics, small molecules and metals. We also show that adding RNA circuitry can invert responses, reduce crosstalk and improve sensitivity without protein engineering. The ROSALIND system can be freeze-dried for easy storage and distribution, and we apply it in the field to test municipal water supplies, demonstrating its potential use for monitoring water quality.

S ynthetic biology is creating new capabilities for engineered biological systems to address pressing global challenges through repurposing, evolving and rewiring the functions of biological components for a range of applications ${ }^{1}$. Among these functions, the ability of cells to use molecular biosensors and genetic networks to sense and respond to changing conditions is being harnessed to create new diagnostic technologies ${ }^{2}$. In particular, cell-free nucleic-acid diagnostics are being developed to detect pathogens and human health biomarkers in complex matrices. These systems use RNA genetic switch design ${ }^{3}$ or CRISPR-mediated interactions ${ }^{4,5}$ to detect specific target DNA or RNA sequences, and trigger gene expression, fluorophore release or other biochemical reactions to produce visual outputs when target molecules are present. The ability to freeze-dry cell-free reaction components for simple storage and distribution has enabled field-deployable technologies for profiling the gut microbiome ${ }^{6}$ and detecting pathogens such as Ebola virus and Zika virus ${ }^{3}$.

It is important to extend the capability of cell-free synthetic biology diagnostics to sense chemical targets other than nucleic acids. This is especially true in the case of water-quality monitoring. Almost $80 \%$ of the world's population is at risk of water insecurity, partly due to a lack of access to clean and safely managed water ${ }^{7}$. Among the many contaminants present in water, some chemical species pose serious threats because they cannot be seen or easily detected. In fact, reliable methods to test for toxic species remain largely constrained to sophisticated, centralized laboratories with expensive instrumentation that requires laboratory technical expertise. These limitations preclude implementation of water-quality testing in the field, most notably in low-resource settings where they are most needed.

To address this need, synthetic biologists have engineered microorganisms to serve as whole cell biosensors (WCBs) ${ }^{8}$. For example, cellular reporter genes can be regulated by allosteric transcription factors (aTFs) that respond to specific chemical contaminants to produce a detectable signal. Changing the aTF allows cellular systems to be engineered to sense a range of contaminants including metals, aromatic compounds and antibiotics ${ }^{8}$. Advantages of this approach include signal amplification through gene expression and selectivity afforded by processes that affect compound bioavailability ${ }^{9}$. However, there are challenges associated with WCBs, including maintenance of viable cells, difficulties with sensing toxic contaminants, membrane transport limitations and the need to address biocontainment and regulatory concerns for deploying living organisms in the field ${ }^{10}$.

In the present study, we sought to preserve the modularity and signal amplification advantages of WCBs, while addressing some of their limitations. We developed cell-free diagnostics using ROSALIND (RNA Output Sensors Activated by Ligand Induction)

'Department of Chemical and Biological Engineering, Northwestern University, Evanston, IL, USA. ${ }^{2}$ Center for Synthetic Biology, Northwestern University, Evanston, IL, USA. ${ }^{3}$ Center for Water Research, Northwestern University, Evanston, IL, USA. ${ }^{4}$ Interdisciplinary Biological Sciences Graduate Program, Northwestern University, Evanston, IL, USA. ${ }^{5}$ Department of Chemistry, Indiana University, Bloomington, IN, USA. ${ }^{6}$ Department of Civil and Environmental Engineering, Northwestern University, Evanston, IL, USA. ' Department of Biological Engineering, Massachusetts Institute of Technology, Cambridge, MA, USA. ${ }^{8}$ Department of Chemical Engineering, Pohang University of Science and Technology, Pohang, Republic of Korea. ${ }^{9}$ Wyss Institute for Biologically Inspired Engineering, Harvard University, Boston, MA, USA. ${ }^{10}$ Departmento de Ingeniería Hidráulica y Ambiental, Pontificia Universidad Católica de Chile, Santiago, Chile. " Centro de Desarrollo Urbano Sustentable, Santiago, Chile. ${ }^{12}$ Department of Geological and Environmental Sciences, California State University, Chico, Chico, CA, USA. ${ }^{13}$ Center for Water and the Environment, California State University, Chico, Chico, CA, USA. ${ }^{14}$ Department of Molecular and Cellular Biochemistry, Indiana University, Bloomington, IN, USA. ${ }^{15}$ Institute for Medical Engineering \& Science, Massachusetts Institute of Technology, Cambridge, MA, USA. ${ }^{16}$ Synthetic Biology Center, Massachusetts Institute of Technology, Cambridge, MA, USA. ${ }^{17}$ Broad Institute of MIT and Harvard, Cambridge, MA, USA. ${ }^{18}$ These authors contributed equally: Jaeyoung K. Jung, Khalid K. Alam. ${ }^{凶}$-mail: jblucks@northwestern.edu 


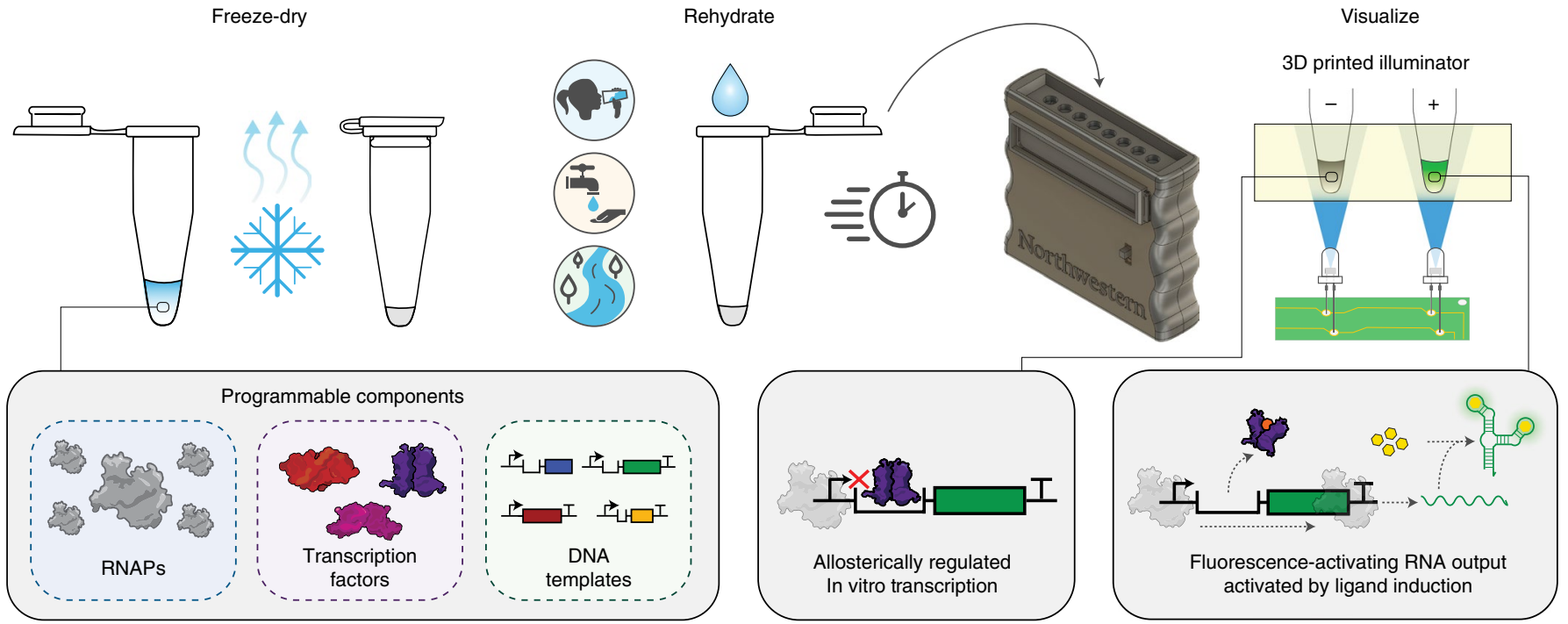

Fig. 1 | The ROSALIND system. The ROSALIND system consists of three programmable components: highly processive RNAPs, aTFs and synthetic DNA transcription templates. Together, these components allosterically regulate the IVT of a fluorescence-activating RNA aptamer; in the absence of an analyte of interest, transcription is blocked, whereas, in its presence, a fluorescent signal is produced. This design is compatible with downstream RNA circuitry that can tune the specificity and sensitivity of transcription factor outputs. ROSALIND can be freeze-dried for field deployment, thereby creating water-quality sensors that are activated on rehydration with the sample of interest. A low-cost, 3D-printed handheld device provides easy visualization of the sensor's RNA output.

to detect contaminants in water (Fig. 1). ROSALIND comprises a highly processive phage RNA polymerase (RNAP), aTFs and engineered DNA transcription templates, which together produce visible outputs on exposure to specific ligands. The use of RNA-level outputs allows signals to be observed within minutes and removes the need for complex and resource-intensive protein translation to detect responses. In addition, the use of in vitro transcription (IVT) reactions removes the need for cells and overcomes biocontainment concerns. ROSALIND represents a capability for simple and rapid field monitoring of chemical species to address growing global health crises in water quality.

\section{Results}

Fluorescent aptamers are rapid visible transcription reporters. We reasoned that fluorescent RNA-level outputs generated through IVT would allow biosensing reactions to be simplified, thereby resulting in faster signal generation than protein-level outputs that must be translated in more complex reaction environments ${ }^{11}$. To demonstrate the viability of fluorescent RNA outputs, we chose the three-way junction dimeric Broccoli $(3 \mathrm{WJdB})$ aptamer $^{12}$ and the fast T7 phage RNAP capable of transcribing 300 nucleotides per second in vitro ${ }^{13}$. DNA transcription templates encoding $3 \mathrm{WJdB}$ behind a T7 RNAP promoter were applied to a commercially available transcription kit supplemented with the Broccoli-binding dye DFHBI-1T (Fig. 2a). Fluorescence activation was monitored in real time and standardized to known amounts of fluorescein (fluorescein isothiocyanate (FITC)) (Supplementary Fig. 1). The IVT reactions generated a fluorescence signal comparable to micromolar equivalent fluorescein (MEF) across a range of input DNA-template concentrations within minutes (Fig. 2b and Supplementary Video 1). Furthermore, the fluorescence activation of $3 \mathrm{WJdB}$ was easily visualized at $0.1 \mathrm{MEF}$ ( $\mu \mathrm{M}$ FITC) with low-cost light-emitting diodes and a stage-lighting filter (Fig. $2 c$ and Supplementary Data 1). In addition, we found that inclusion of a T7 terminator in the transcription templates resulted in improved fluorescence activation (Supplementary Fig. 2).

We next compared the fluorescence intensity and kinetics of the RNA output with that of superfolder green fluorescent protein
(sfGFP $)^{14}$ produced using a commercial kit based on the PURE system $^{15}$. 3WJdB-expressing IVT reactions and sfGFP-expressing PURE reactions were run using equimolar amounts of DNA templates. In this setup, the RNA output immediately began to increase in fluorescence intensity, whereas sfGFP required at least $9 \mathrm{~min}$ before a detectable signal was observed (Fig. 2d and Supplementary Fig. 3). In addition, over the 4-h time course, $3 \mathrm{WJdB}$ transcription produced substantially more fluorescence than sfGFP (Fig. 2d and Supplementary Fig. 3), and the signal generated with a homemade transcription reaction was comparable to that of the commercial kit (Extended Data Fig. 1).

Together, these results show that IVT of fluorescence-activating RNA aptamers can be repressed by aTFs and induced by targets of interest, thereby establishing a proof of concept for the ROSALIND system.

aTFs enable ligand responsiveness. We next sought to create a ligand-responsive biosensor by using an aTF to regulate T7 RNAP IVT. We reasoned that the binding of an aTF to its cognate operator sequence would prevent T7 RNAP from promoter escape in the absence of ligand, and ligand-mediated de-repression of an aTF would result in a fluorescence signal (Fig. 1).

We first investigated how aTF-operator interactions can block T7 RNAP transcription in the absence of ligand. We chose TetR, a well-characterized model aTF that binds the 19-base pair (bp) palindromic tet operator sequence (tet $O$ ) in the absence of its cognate ligand tetracycline ${ }^{16}$. We designed a series of transcription templates to explore the effect of spacing between the minimal 17-bp T7 promoter and the tet $O$ sequence (Fig. 2e). We used the native sequence that follows the canonical T7 RNAP promoter ${ }^{17}$ as a spacer in 2-bp increments from 0 to $14 \mathrm{bp}$, immediately followed by tet $O, 3 \mathrm{WJdB}$ and T7 terminator sequences. IVT reactions were set up as before using homemade reactions, with the addition of purified recombinant TetR protein in 100-fold excess of the DNA template. We observed that the absence of any spacing resulted in no fluorescence activation in either the presence (regulated) or the absence (unregulated) of TetR (Fig. 2f). However, robust fluorescence activation was observed without TetR when using a 2-6-bp spacer, which was reduced to baseline levels when regulated by TetR. Spacers 


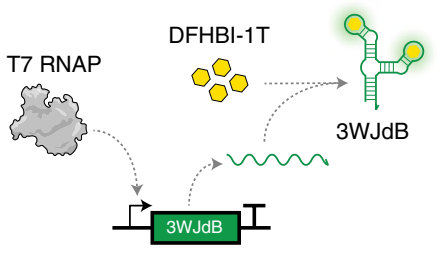

Linear dsDNA transcription template

e

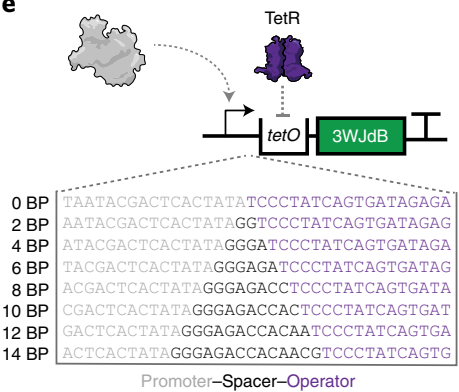

b

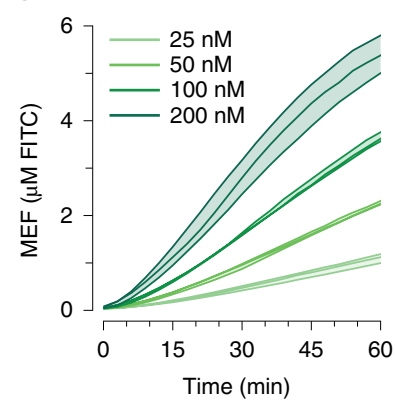

f

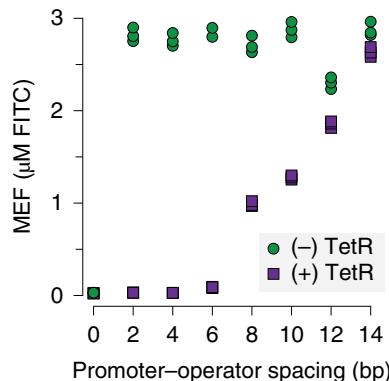

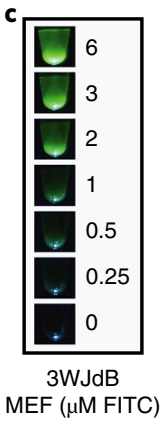

d

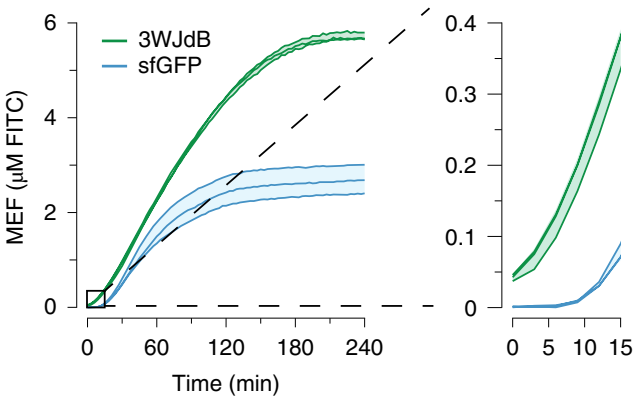

g

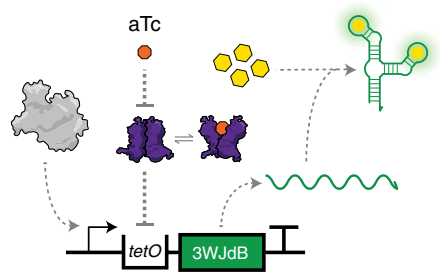

h

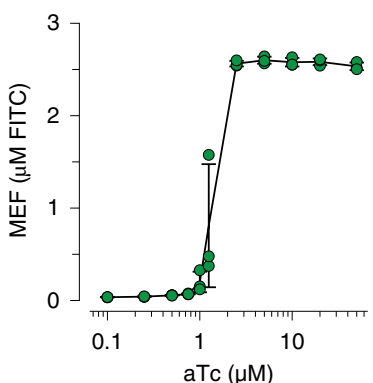

Fig. 2 | IVT of a fluorescence-activating aptamer rapidly generates a visible RNA output that can be allosterically regulated. a, T7 RNAP transcription of the three-way junction dimeric Broccoli (3WJdB) aptamer. Binding of DFHBI-1T by 3WJdB activates its fluorescence. $\mathbf{b}$, Titration of the linear double-stranded DNA template in a commercial transcription kit generates fluorescence signal comparable to MEF when standardized to soluble fluorescein ( $\mu \mathrm{M}$ FITC). c, Visual detection of $3 \mathrm{WJdB}$ :DFHBI-1T fluorescence, with values corresponding to the MEF of soluble FITC. The results are shown for one representative of $n=3$ independent biological replicates. d, Comparison of fluorescence kinetics of 3WJdB synthesized from IVT, and sfGFP synthesized from in vitro transcription-translation, using an equimolar DNA template ( $50 \mathrm{nM})$. e, IVTs can be allosterically regulated with a template configured to bind a purified transcription factor (TetR) via tetO placed downstream of the T7 promoter. A series of spacers in 2-bp intervals was constructed to evaluate the impact of spacer length on the ability to regulate transcription. $\mathbf{f}$, End-point data (at $4 \mathrm{~h}$ ) shown for promoter-operator spacer variants regulated (with $2.5 \mu \mathrm{M}$ TetR dimer, $25 \mathrm{nM}$ DNA template) and unregulated (without TetR). $\mathbf{g}$, Induction of a TetR-regulated IVT reaction occurs in the presence of the cognate ligand, aTc, which binds to TetR and prevents its binding to tetO. This allows transcription to proceed, leading to fluorescence activation. $\mathbf{h}$, Dose response with aTc, measured at 240 min with $25 \mathrm{nM}$ DNA template and $1.25 \mu \mathrm{M}$ TetR dimer. All data shown are for $n=3$ independent biological replicates as lines $(\mathbf{b}, \mathbf{d})$ or points $(\mathbf{f}, \mathbf{h})$ with raw fluorescence values standardized to MEF ( $\mu$ M FITC). Shading (b,d) and error bars (h) indicate the average value of three independent biological replicates \pm s.d. The original, uncropped image shown in $\mathbf{c}$ can be found in Supplementary Data 1. dsDNA, double-stranded DNA.

that were $\geq 8 \mathrm{bp}$ resulted in fluorescence activation in the regulated reactions. We therefore chose the shorter functional spacers for subsequent promoter-operator designs.

We next sought to determine whether TetR can be de-repressed with a cognate ligand to permit IVT and therefore act as a biosensor (Fig. 2g). We first performed IVT reactions over a range of TetR concentrations using the transcription template with a 4-bp spacer (Extended Data Fig. 2). We found that a 25-fold excess of TetR dimer to transcription template was the minimal amount of repressor needed to ensure tight regulation of transcription, whereas a 50 -fold excess of TetR dimer performed similarly to the 100-fold excess (Extended Data Fig. 2). We then set up IVT reactions using these TetR and DNA template concentrations and included a range of anhydrotetracycline ( $\mathrm{aTc}$ ) concentrations. We observed strong repression over $4 \mathrm{~h}$ through high nanomolar amounts of aTc, with half-maximal induction between 1 and $2.5 \mu \mathrm{M}$ aTc (Fig. $2 \mathrm{~h}$ and Extended Data Fig. 3). Importantly, when induced with $2.5 \mu \mathrm{M}$ aTc, the kinetics of induction follow those of the unregulated transcription reaction (Extended Data Fig. 2).

Together, these results show that aTF-regulated IVT of fluorescence-activating RNA aptamers can serve as biosensors, establishing a proof-of-concept basis for the ROSALIND system.

Modularity allows sensing of organic molecules and metals. Next, we sought to expand the range of molecules that can be detected with ROSALIND by leveraging the natural diversity of $\mathrm{aTFs}^{18}$. Inspired by earlier work with $\mathrm{WCBs}^{8}$, we reasoned that ROSALIND could sense a wide range of important targets by changing the operator sequence within the transcription templates and including the corresponding aTF in the reaction (Extended Data Fig. 2 and Supplementary Data 2).

We began with antibiotics, the overuse of which is resulting in the contamination of freshwater supplies and driving antimicrobial resistance $^{19}$. We first tested our TetR-based sensor to detect tetracycline (Fig. 3a and Extended Data Fig. 4a) and the semisynthetic antibiotic doxycycline (Fig. 3b and Extended Data Fig. 4b), both of which are on the World Health Organization's (WHO's) list of essential medicines. Reactions with TetR-regulated transcription templates and purified TetR were run using either laboratory-grade water $(-)$ or similar water with added antibiotic $(+)$. In both cases, we observed generation of fluorescence with the antibiotic present and no fluorescence in the water control.

We then leveraged $O \operatorname{trR}^{20}$, a member of the multiple antibiotic-resistance repressor (MarR) family of $\mathrm{aTFs}^{21}$, to serve as a biosensor for oxytetracycline (Fig. 3c and Extended Data Fig. 4c). To expand the range of tetracycline antibiotic sensing further, we identified a putative MarR-family aTF analogous to OtrR, CtcS ${ }^{22}$, and the divergent operon regulated by CtcS, and designed a minimal operator sequence to enable its use in ROSALIND for sensing chlortetracycline (Fig. 3d and Extended Data Fig. 4d). 

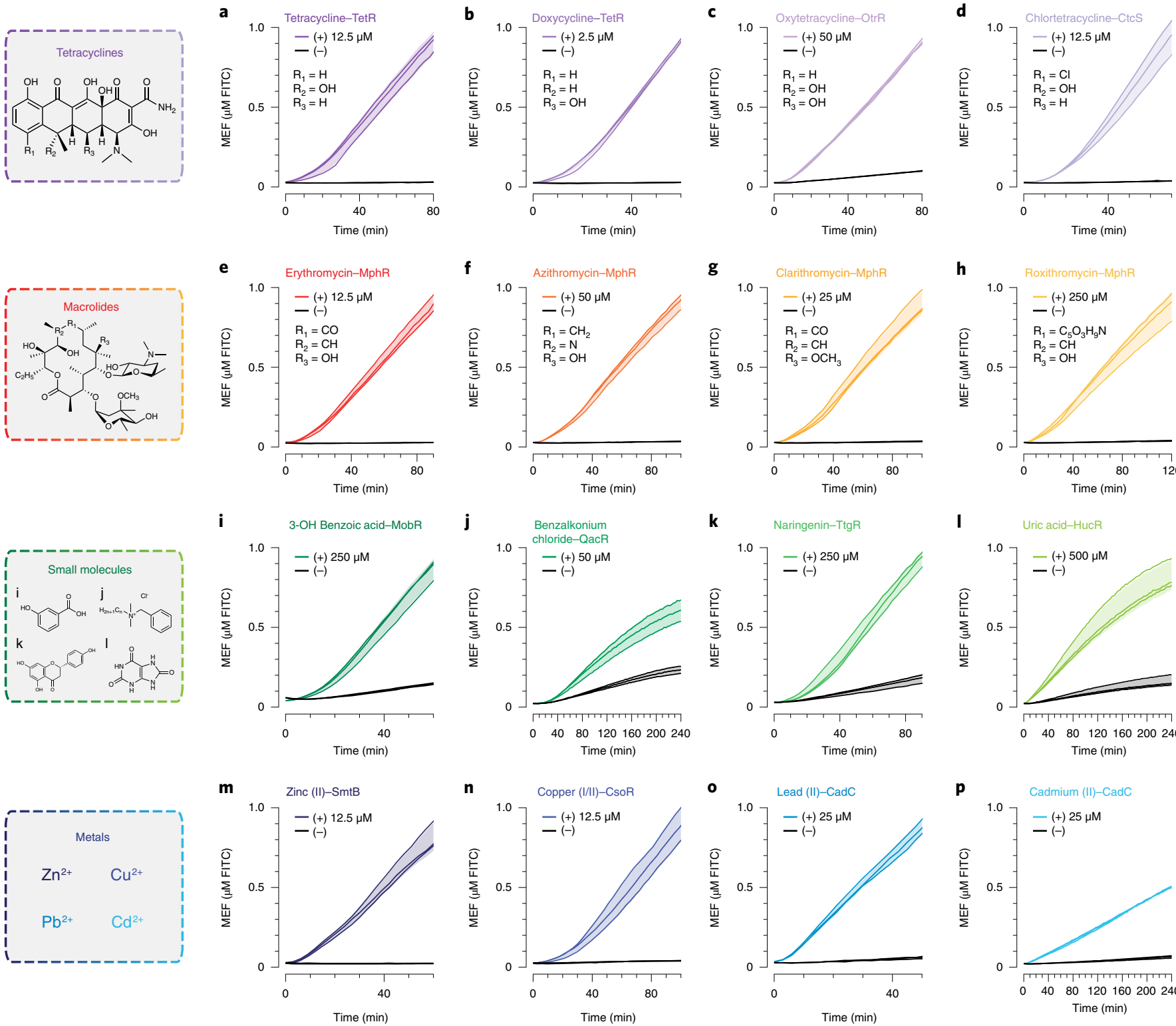
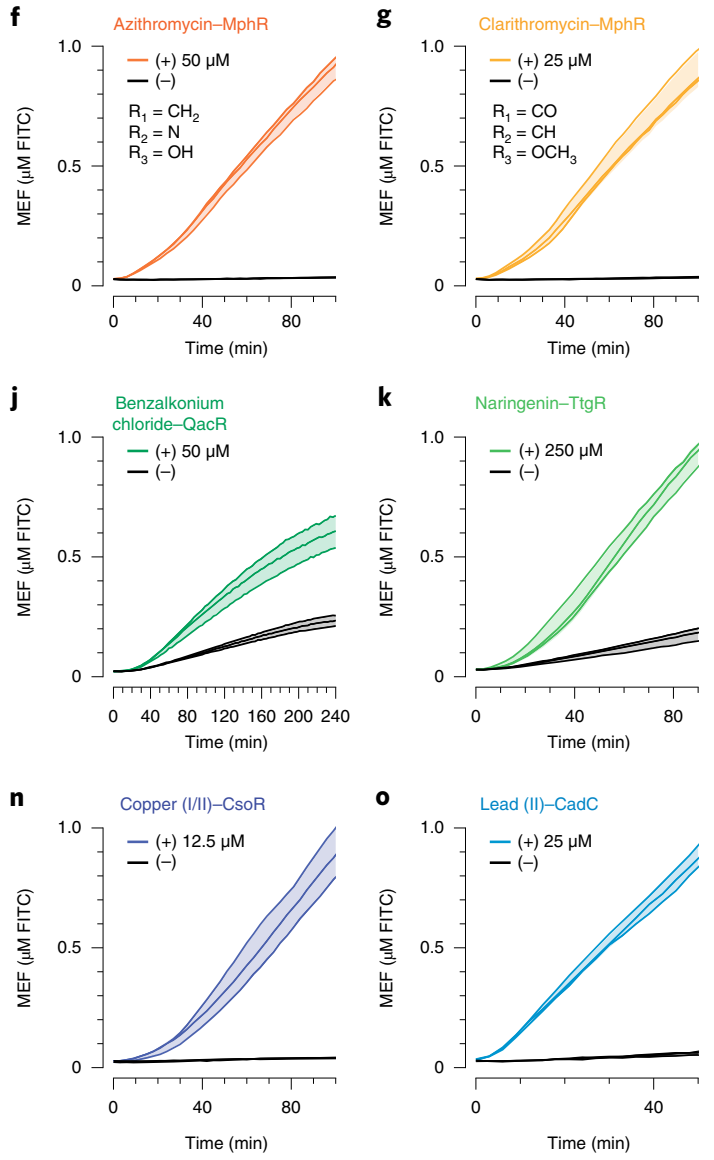
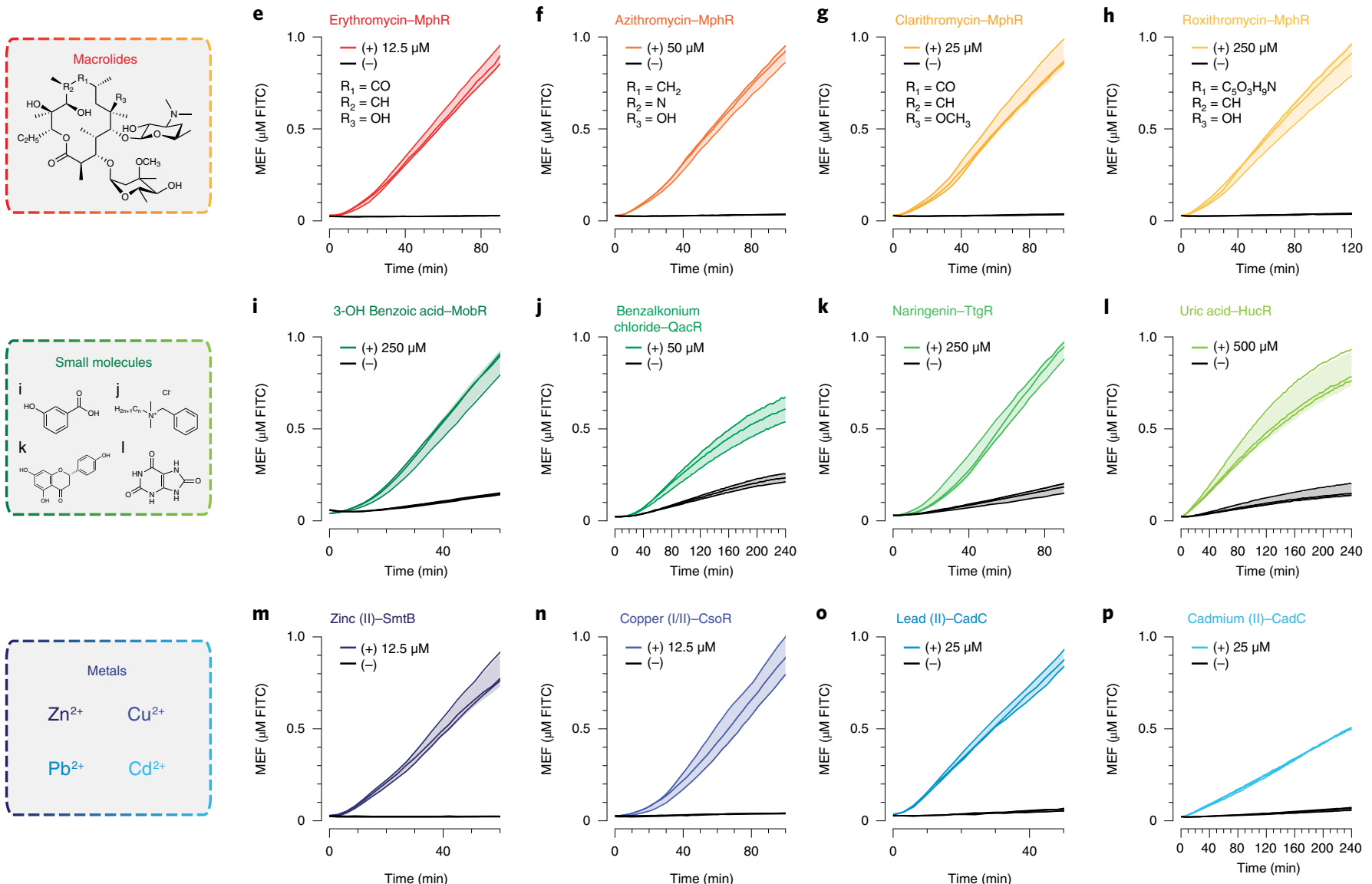

Fig. 3 | ROSALIND is modular and can be used to sense complex molecules, small molecules and metal ions. a,b, TetR can be used to sense tetracycline (a), doxycycline (b) and other tetracycline-family antibiotics. c, Oxytetracycline sensing with OtrR. $\mathbf{d}$, Chlortetracycline sensing with CtcS. e-h, MphR senses the macrolides erythromycin (e), azithromycin (f), clarithromycin ( $\mathbf{g}$ ) and roxithromycin (h). $\mathbf{i}$, 3-Hydroxybenzoic acid sensing with MobR. j, Benzalkonium chloride sensing with QacR. k, Naringenin sensing with TtgR. I, Uric acid sensing with HucR. m, $\mathrm{Zn}^{2+}$ sensing from $\mathrm{ZnSO}_{4}$ with $\mathrm{SmtB}_{\text {. }}$ n, $\mathrm{Cu}^{2+} / \mathrm{Cu}^{+}$sensing from $\mathrm{CuSO}_{4}$ with $\mathrm{CsoR}$ from Bacillus subtilis. o,p, $\mathrm{Pb}^{2+}$ sensing from $\mathrm{PbCl}_{2}(\mathbf{0})$ and $\mathrm{Cd}^{2+}$ sensing from $\mathrm{CdCl}{ }_{2}$ with $\mathrm{CadC}$ (p). Each reaction contains the indicated amount of ligand (+) dissolved in laboratory-grade water, ethanol, Tris-based buffer, dimethylsulfoxide or diluted sodium hydroxide, or a laboratory-grade water control (-) (Supplementary Table 2). All data shown are for $n=3$ independent biological replicates as lines with raw fluorescence values standardized to $\operatorname{MEF}(\mu M$ FITC). Shading indicates the average value of three independent biological replicates \pm s.d. DNA template and aTF concentrations used in each reaction can be found in Supplementary Table 2.

We next sought to expand sensing to the macrolide class of antibiotics that are also widely used in human health. We chose a TetR-family aTF, MphR ${ }^{23}$, to detect erythromycin, a drinking water contaminant of emerging concern ${ }^{24}$, azithromycin, clarithromycin and roxithromycin (Fig. 3e-h and Extended Data Fig. 4e-h). We note that this observed broad range of MphR specificity is expected based on its known promiscuity, and is probably a common feature of xenobiotic-sensing aTFs ${ }^{25}$.

After demonstrating the ability of ROSALIND to detect complex antibiotic molecules, we sought to leverage the system for the detection of other organic molecules, focusing on those with public health relevance. In particular, there has been a growing concern that unregulated contaminants, such as those generated from the large use of pharmaceuticals and personal care products, interfere with or are unaffected by water treatment, and are causing widespread health and environmental concerns ${ }^{26}$. We started with the perfume additive 3-hydroxybenzoic acid as a model pharmaceuti$\mathrm{cal}$ and personal care product that can de-repress the Comamonas testosteroni KH122-3S MarR-family aTF, MobR ${ }^{27}$. When ROSALIND reactions were configured with MobR and its corresponding DNA template, we observed strong activation of fluorescence only in the presence of 3-hydroxybenzoic acid (Fig. 3i and Extended Data Fig. 4i). We further demonstrated that ROSALIND could be used to sense benzalkonium chloride, a widely used 
disinfectant that can promote antimicrobial resistance ${ }^{28}$, by leveraging the Staphylococcus aureus TetR-family aTF, QacR ${ }^{29}$ (Fig. 3j and Extended Data Fig. 4j).

Some organic molecules such as naringenin ${ }^{30}$ and uric acid can serve as important markers for human health and nutrition. Using the Pseudomonas putida TetR-family aTF, $\operatorname{TtgR}^{31}$, and an optimized $\operatorname{ttg} O$ variant sequence ${ }^{32}$, we designed ROSALIND reactions to sense naringenin in the tens of micromolar range (Fig. 3k and Extended Data Fig. 4k). In addition, we sought to detect uric acid, an early indicator of gout when present at $\geq 6 \mathrm{mg} \mathrm{dl}^{-1}$ within bodily fluids ${ }^{33}$. By incorporating HucR, a MarR-family aTF from Deinococcus radiodurans ${ }^{34}$, and the corresponding DNA template, we were able to detect uric acid within water samples at approximately $50 \mu \mathrm{M}$ $\left(\sim 0.84 \mathrm{mg} \mathrm{dl}^{-1}\right)$ or higher (Fig. 31 and Extended Data Fig. 4l).

Among water contaminants, metals have become a major issue due to aging infrastructure, mining operations and industrial waste disposal, and from natural abundance ${ }^{35-37}$. To enable metal detection using ROSALIND, we first identified the canonical zinc-sensing aTF, SmtB, from the ArsR/SmtB family of regulators ${ }^{38}$. Using SmtB and the corresponding DNA template, we were able to sense $\mathrm{Zn}$ (II) at $\geq 2.5 \mu \mathrm{M}(\geq 163 \mathrm{ppb})$, which is below the US Environmental Protection Agency's (EPA's) secondary drinking guideline of $5 \mathrm{ppm}$ (Fig. $3 \mathrm{~m}$, Extended Data Fig. $4 \mathrm{~m}$ and Supplementary Table 1).

Although many metals are toxic at high concentrations, of particular concern are copper and lead ${ }^{36,37}$ due to toxic effects such as psychiatric disorders, impaired neural development and organ damage. As such, their levels are regulated in drinking water by the US EPA's Lead and Copper Rule (LCR). Among the many copper-sensing aTFs is CsoR, a Cu(I)-sensing aTF first identified in Mycobacterium tuberculosis that forms a homotetramer to bind four copper ions ${ }^{39}$. When configured into ROSALIND with the appropriate DNA template, our sensor was able to detect copper at $\geq 5 \mu \mathrm{M}$ ( $\geq 318 \mathrm{ppb})$ (Fig. 3n and Extended Data Fig. 4n). It is of interest that, although the cognate ligand for $\mathrm{CsoR}$ is $\mathrm{Cu}(\mathrm{I})$, we observed that the reaction senses $\mathrm{Cu}$ (II) from added $\mathrm{CuSO}_{4}$, possibly due to the presence of $10 \mathrm{mM}$ dithiothreitol (DTT) in IVT reactions that can reduce $\mathrm{Cu}$ (II) to $\mathrm{Cu}(\mathrm{I})$. Another ArsR/SmtB family aTF, CadC, is found in pI258 of $S$. aureus and is known to bind a number of metals, including lead and cadmium ${ }^{40}$. When implemented in ROSALIND with appropriately configured DNA templates, we could robustly detect both lead and cadmium at $\geq 1.25 \mu \mathrm{M}$ ( $\geq 259 \mathrm{ppb}$ for lead and $\geq 141 \mathrm{ppb}$ for cadmium) (Fig. 3o,p and Extended Data Fig. 4o,p).

In total, we employed 11 different aTFs to sense 16 different ligands that are structurally and functionally diverse. An investigation into their limits of detection (Extended Data Fig. 4 and Supplementary Data 3) showed that the majority detect their respective ligands in the micromolar range. In addition, characterization of the inhibitory effects of the ligands and the solvents used to prepare them (Supplementary Fig. 4 and Supplementary Table 2) suggests that ROSALIND can tolerate much higher concentrations of the ligands than their respective WHO/EPA-regulated limits (Supplementary Table 1).

RNA circuitry can invert logic and fix crosstalk. Specificity is a key metric for the performance of biosensors. In some cases, a single aTF is well known to sense multiple ligands, as is the case with TetR sensing tetracyclines, MphR sensing macrolides and CadC sensing both lead and cadmium ${ }^{18,25,40}$. In these cases, ROSALIND is best viewed as reporting on the presence of a specified family of stereochemically related ligands. To investigate whether ROSALIND reactions are specific for the indicated compounds outside of these known cases, we tested ROSALIND reactions configured with TetR, MphR, MobR, QacR, TtgR, HucR, SmtB, CsoR or CadC, along with their corresponding DNA templates, with a panel of cognate ligands. Tetracycline, erythromycin and lead were chosen as representative members of each class of compounds for TetR, MphR and CadC, respectively. In general, we found strong signals from cognate ligands with minimal crosstalk, indicating that ROSALIND reactions are generally specific for their intended ligands when DNA template and aTF concentrations are optimized (Fig. 4a and Extended Data Fig. 5).

We did, however, find significant crosstalk from the CsoR-containing copper sensor when induced with zinc (Fig. 4a and Extended Data Fig. 5). Notably there was no corresponding crosstalk across $\mathrm{SmtB}$, the zinc sensor and copper. This created the opportunity to construct a sensor by combining the activity profiles of CsoR and SmtB in a single reaction to fix the crosstalk of CsoR. Specifically, this sensor was configured based on a Cu NIMPLY Zn ( $\mathrm{Cu}$ AND NOT $\mathrm{Zn}$ ) circuit that would respond only to copper and not zinc. Implementation of this circuit requires the presence of zinc to inhibit the $3 \mathrm{WJdB}$ signal produced by CsoR.

To achieve this zinc-mediated inhibition, we leveraged the recent development of RNA kleptamers-short antisense RNAs designed to inhibit Broccoli by disrupting the aptamer folding that enables fluorescence activation ${ }^{41}$. We found that fluorescence activation of $3 \mathrm{WJdB}$ could be inhibited through unregulated IVT of the KB2 kleptamer with T7 RNAP (Extended Data Fig. 6a), and that KB2 transcription could be regulated by TetR, by including the tet $O$ sequence in the KB2 template (Extended Data Fig. 6b). Addition of its cognate ligand, aTc, effectively de-repressed TetR from the KB2 template, thereby allowing KB2 transcription and inverting the output of the TetR sensor (Extended Data Fig. 6c). We also demonstrated the generality of the kleptamer inversion strategy using the aporepressor AdcR from Streptococcus pneumoniae ${ }^{21,42}$, which binds its operator in the presence of $\mathrm{Zn}$ (II), and inverting it into a zinc-activated sensor (Extended Data Fig. 6d).

We used the kleptamer strategy within the Cu NIMPLY Zn circuit. In this circuit design, the transcription of $3 \mathrm{WJdB}$ is regulated by CsoR, whereas the transcription of KB2 is regulated by SmtB (Fig. 4b). In the presence of copper only, CsoR is de-repressed from the $3 \mathrm{WJdB}$ transcription template, generating $3 \mathrm{WJdB}$. In the presence of zinc only, both CsoR and SmtB are de-repressed from their respective transcription templates, generating $3 \mathrm{WJdB}$ and $\mathrm{KB} 2$, which is then able to prevent $3 \mathrm{WJdB}$ from binding its dye, shutting off the fluorescence signal. When implemented in ROSALIND, the $\mathrm{Cu}$ NIMPLY $\mathrm{Zn}$ circuit performed as expected, correcting the crosstalk of CsoR with zinc (Fig. 4c). Furthermore, the circuit was able to fix the crosstalk across a wide range of zinc concentrations, while keeping the dose-response curves against copper unaffected (Supplementary Fig. 5). Overall, these results show that RNA genetic circuitry can be designed to address issues with aTF specificity without having to use protein engineering strategies.

Sensitivity can be tuned with transcriptional circuitry. A shortcoming of our approach is that it is limited by the natural sensitivity of the aTF employed in the ROSALIND reaction. For example, our TetR sensor was activated by doxycycline at $1.25 \mu \mathrm{M}(556 \mathrm{ppb})$, whereas the typical concentrations of antibiotic pollution found in environmental water samples are in the range of tens of parts per billion $^{43}$. We therefore sought to tune the sensitivity of ROSALIND reactions using a generalizable strategy that did not require engineering of the aTF.

As proof-of-concept demonstrations, we first sought to tune the copper- and lead-sensing reactions containing CsoR and CadC, respectively. The dose-response curves of the copper and lead sensors indicate that the limit of detection for copper $(0.3 \mathrm{ppm}$ of $\mathrm{Cu}(\mathrm{II}))$ is below the threshold set by the US EPA's LCR (1.3 ppm), whereas that of lead $(259 \mathrm{ppb})$ is an order of magnitude above the desired threshold (15 ppb) (Extended Data Fig. 7 and Supplementary Table 1). To desensitize the copper sensor, we increased the CsoR concentration to allow any excess to act as a copper chelator and shifted the dose-response curve to activate near the desired LCR threshold 


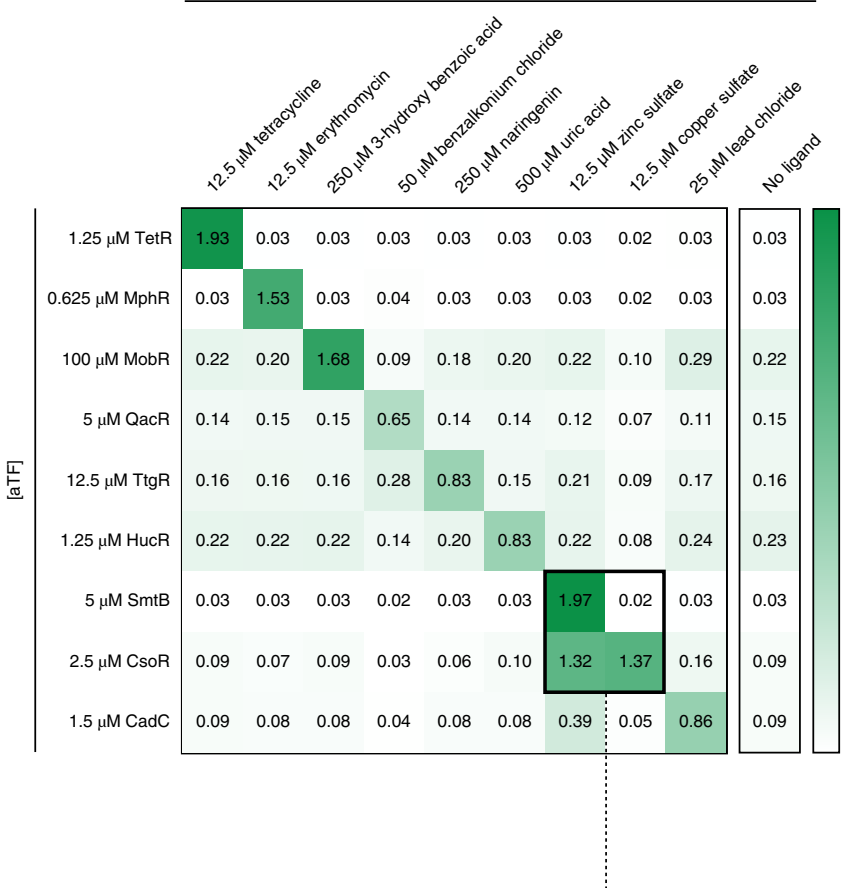

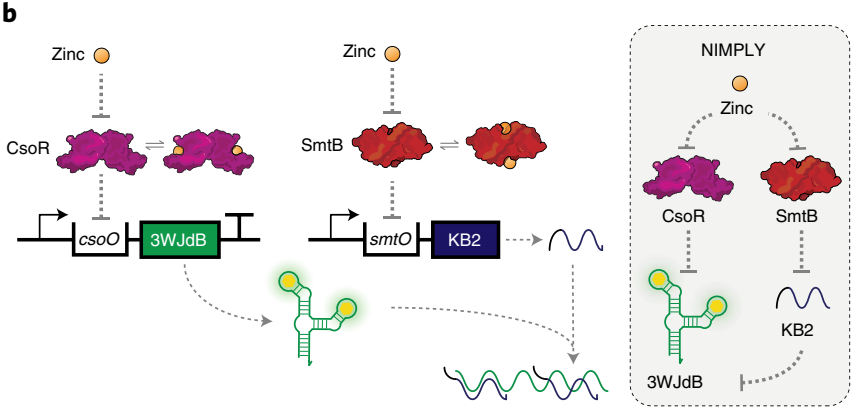
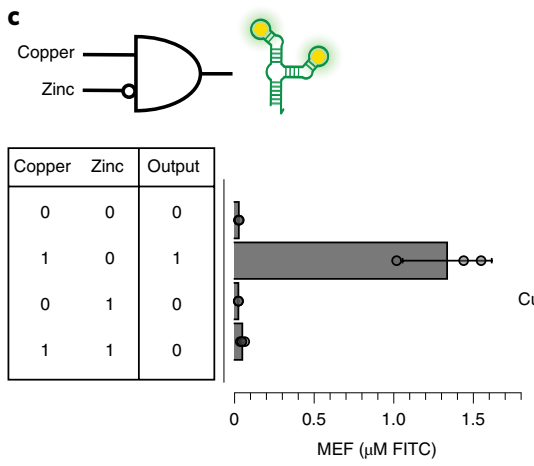

NIMPLY applied

Fig. 4 | Crossreactivity of ROSALIND reactions can be corrected using an RNA logic circuit. a, A subset of ROSALIND reactions was tested to evaluate crossreactivity with different ligands. Significant crosstalk was observed from the copper-sensing aTF, CsoR, where the sensor detects copper as well as zinc. The values on the heatmap represent the average MEF ( $\mu$ M FITC) of $n=3$ independent biological replicates after 240 min (see Extended Data Fig. 5 for all data). DNA templates used for reactions were $25 \mathrm{nM}$ for TetR, MphR, SmtB and CsoR; $20 \mathrm{nM}$ for QacR; $12.5 \mathrm{nM}$ for HucR; $10 \mathrm{nM}$ for CadC; and $5 \mathrm{nM}$ for MobR and TtgR. b, To fix the crossreactivity of CsoR, a Cu NIMPLY Zn logic gate was implemented consisting of the CsoR-controlled 3WJdB template and a SmtB-controlled template encoding a $3 \mathrm{WJdB}$-interfering kleptamer (KB2). In the presence of zinc, the fluorescence of $3 \mathrm{WJdB}$ is inhibited by the presence of KB2, whereas, in the presence of copper alone, only $3 \mathrm{WJdB}$ is produced. $\mathbf{c}$, When implemented in ROSALIND, the Cu NIMPLY Zn circuit fixes the crossreactivity of the copper sensor. Each reaction contained $25 \mathrm{nM} 3 \mathrm{WJdB}$ template, $150 \mathrm{nM}$ KB2 template, $2.5 \mu \mathrm{M}$ CsoR tetramer and $5 \mu \mathrm{M}$ SmtB dimer. The concentrations of copper and zinc tested in the bar plot are $10 \mu \mathrm{M}$ each. The bar plot in c shows data for $n=3$ independent biological replicates as points, with raw fluorescence values standardized to MEF ( $\mu$ M FITC), and bars representing averages of the replicates. Error bars indicate the average values of three independent biological replicates \pm s.d. The matrix in c shows the average MEF ( $\mu$ M FITC) of $n=3$ independent biological replicates after 240 min. The aTF concentrations indicated in $\mathbf{a}$ and $\mathbf{c}$ are dimer concentrations except for CsoR, which is a tetramer.

of $1.3 \mathrm{ppm}$ of copper from added $\mathrm{CuSO}_{4}$ (Extended Data Fig. 7a). To sensitize the lead sensor, we thus employed the converse of our CsoR strategy. By lowering the CadC dimer concentration to $0.5 \mu \mathrm{M}$, we were able to shift the dose-response curve to be more sensitive to detect tens of parts per billion of $\mathrm{Pb}$ (II) (Extended Data Fig. 7b). However, this strategy resulted in a significant increase in signal in the absence of lead.

To address this limitation, we sought to implement a different method to improve sensor sensitivity, based on the previous development of a TetR-binding RNA aptamer (anti-TetR) which de-represses TetR by mimicking its operator binding site tet $O\left(\right.$ ref. $\left.^{44}\right)$. Taking advantage of this feature of anti-TetR, we implemented an RNA-feedback loop designed to enhance the expression of $3 \mathrm{WJdB}$ at low concentrations of doxycycline by regulating anti-TetR transcription with TetR (Fig. 5a). As configured, when doxycycline is present, the circuit is designed to de-repress TetR and initiate the expression of $3 \mathrm{WJdB}$ as well as anti-TetR. Once transcribed, anti-TetR can then further de-repress any TetR that is blocking the transcription of $3 \mathrm{WJdB}$. When implemented in ROSALIND, the feedback circuit improved the sensitivity of the doxycycline sensor by an order of magnitude to the $\sim 0.2-\mu \mathrm{M}(89 \mathrm{ppb}$ ) range (Fig. $5 \mathrm{~b}$ and Supplementary Data 3). These results demonstrate that TetR-based sensors can be sensitized to the ranges of antibiotic concentrations typically found in the environment, although preconcentration strategies would further enhance this. Moreover, this demonstrates that ROSALIND is compatible with RNA-feedback circuits that can improve the sensitivity of aTFs without any protein engineering.

Application to municipal water samples. Next, we examined whether ROSALIND can be formulated for distribution in the field. Many of the advances in synthetic biology diagnostic technologies have been enabled through freeze-drying cell-free systems for convenient storage and distribution before rehydration at the point of use $^{3,45}$. To test whether the ROSALIND system can be freeze-dried, we explored a range of known lyoprotectants and determined that nonreducing disaccharides, such as sucrose and trehalose, in combination with a sugar alcohol, mannitol, provided substantial lyoprotection. In addition, we developed a freeze-drying and packaging procedure (Supplementary Video 2 ) that enabled freeze-dried ROSALIND reactions to be stored at ambient temperature for at least 2.5 months and remain functional, although with some impact on measured fluorescence (Extended Data Fig. 8).

In addition to freeze-drying, field deployment also requires a convenient approach to detecting the fluorescence signals generated by ROSALIND. To this end, we developed a three-dimensional (3D)-printed, handheld device that could visualize fluorescent output using the naked eye (Extended Data Fig. 9 and Supplementary Data 4). The use of off-the-shelf components assembled onto a 

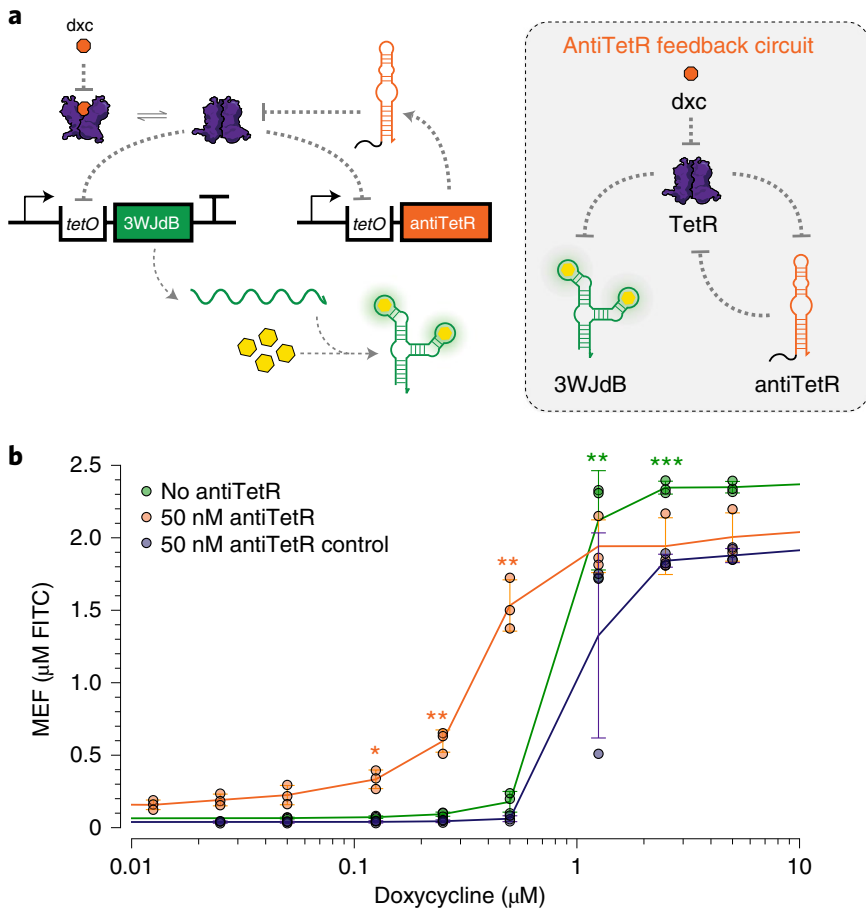

Fig. 5 | Sensitivity of ROSALIND reactions can be tuned using an RNA-feedback circuit. a, A TetR-binding RNA, anti-TetR, can be used to improve the limit of detection of the TetR sensor. In this feedback circuit, TetR regulates both the expression of $3 \mathrm{WJdB}$ and anti-TetR. In the presence of the cognate ligand, doxycycline, anti-TetR is transcribed and further de-represses TetR to enhance $3 \mathrm{WJdB}$ expression. $\mathbf{b}$, When implemented in ROSALIND, the anti-TetR feedback circuit improves the sensitivity of TetR by an order of magnitude. Each reaction contains $25 \mathrm{nM} 3 \mathrm{WJdB}$ template, $50 \mathrm{nM}$ anti-TetR template and $1.25 \mu \mathrm{M}$ TetR dimer. A transcription template encoding the reverse sequence of anti-TetR was used as a control to demonstrate that sensitization required the anti-TetR interaction. All data shown are for $n=3$ independent biological replicates as points with raw fluorescence values standardized to MEF ( $\mu$ M FITC). Error bars indicate the average values of three independent biological replicates \pm s.d. The ligand concentrations at which the signal was distinguishable from the background were determined using a two-tailed, heteroscedastic Student's $t$-test against the no-ligand condition, and their $P$ value ranges are indicated with asterisks ( $\left.{ }^{\star \star \star} P<0.001,{ }^{\star \star} P=0.001-0.01,{ }^{\star} P=0.01-0.05\right)$. Exact $P$ values along with degrees of freedom can be found in Supplementary Data 3. Data for no-ligand condition were excluded in $\mathbf{b}$ because the $x$ axis is on the log scale, and are presented in Supplementary Data 3.

printed circuit board allowed us to optimize the device for visualization of $3 \mathrm{WJdB}$ fluorescence using blue light for fluorescence excitation and yellow-stage light film as a long-pass optical filter.

With these results, we next sought to demonstrate a field application of ROSALIND to address a water-quality issue of growing concern-the contamination of municipal water supplies with metals. We studied the influence that the 2018 Camp Fire in Paradise, CA, had on its water distribution system. Preliminary field sampling of unused Paradise municipal water fountains during the cleanup efforts in the spring of 2019 suggested elevated levels of both copper and zinc. We therefore sought to field test our copper (CsoR), zinc (SmtB) and $\mathrm{Cu}$ NIMPLY Zn sensors in the context of Paradise municipal water.

We first confirmed that this set of ROSALIND reactions functioned correctly when municipal drinking water collected in Evanston, IL, spiked with either copper or zinc, was used for rehydration (Supplementary Fig. 6). Sensors were then manufactured, arrayed in strip tubes, freeze-dried, packaged in Evanston and shipped overnight to Chico, CA, at ambient temperature (Fig. 6a). We included control reactions for rehydration with laboratory-grade, metal-free water to confirm reactions performed as intended and to provide a convenient visual comparison against sampled water by eye (Fig. 6b). On receipt in California, we sampled and filtered water from four different municipal locations in Paradise ${ }^{46}$. The shipped sensors were rehydrated with these samples or laboratory-grade, metal-free water and placed in a portable incubator at $37^{\circ} \mathrm{C}$ for $4 \mathrm{~h}$. Photographs were taken of the reactions placed in our handheld illuminator after $2 \mathrm{~h}$ and overnight (Fig. 6c-f). Rehydrated reactions were then shipped back to Evanston for plate-reader quantification (Fig. 6c-f), which was possible due to the observed stability of fluorescence signals from ROSALIND reactions once complete (Extended Data Fig. 8a). Of the four sites sampled, two contained zinc only and two contained both zinc and copper, as verified using flame atomic absorption spectroscopy (FAAS) (Supplementary Fig. 7). In all cases, the freeze-dried, shipped and applied reactions behaved as expected (Fig. 6c-f). To further demonstrate ROSALIND functionality in complex matrices, additional laboratory tests of freeze-dried copper sensors were performed against Lake Michigan water spiked with copper as well as surface waters from Chile, known to be highly enriched with copper from a combination of geogenic and anthropogenic processes ${ }^{47}$. We confirmed that the sensors can function with environmental water samples as well, although with some inhibition of the fluorescence signal (Extended Data Fig. 10).

Overall, these results demonstrate that ROSALIND can function in the field to detect water contaminants of interest and, as configured, are best suited to indications of presence/absence at specific thresholds.

\section{Discussion}

In the present study, we report the development of a cell-free biosensor platform, ROSALIND, for detection of a wide range of chemical compounds and elements in water. ROSALIND consists of simple and defined aTF-regulated IVT reactions that produce RNA outputs as a function of ligand concentration. Inspired by previous WCB approaches ${ }^{8}$, this modular architecture allowed us to develop 16 different sensors by changing the aTF-operator pair (Fig. 3). The use of fast phage polymerases generated visible signals to the naked eye in less than an hour. In addition, the reactions and handheld illuminators could be manufactured inexpensively (Supplementary Table 3 and Supplementary Data 4).

A key advantage of transcriptional RNA outputs was our ability to design RNA-level circuits to address well-known shortcomings of aTFs without performing any protein engineering. In particular, we were able to demonstrate that aTF-ligand crosstalk can be eliminated with RNA-level logic gates (Fig. 4), and aTF sensitivity can be improved through an RNA-mediated feedback circuit (Fig. 5). We anticipate that these RNA-level circuit strategies will be applicable to optimizing broad classes of aTFs.

The simple and defined nature of ROSALIND makes it amenable to extensions. For example, multiplexing can be added with additional fluorescent aptamers ${ }^{48}$, and further engineering of the aTF or operator sequences can be used to enhance sensitivity, modify specificity and otherwise tune system performance ${ }^{49}$. In addition, other uses besides water contaminant detection are possible, including the high-throughput screening of poorly characterized aTFs to uncover their cognate ligands and putative operator sequences, as we showed with our validation of the putative aTF, CtcS (Fig. 3d).

A key limitation of ROSALIND is that it is currently limited to aTFs that block RNAP elongation unless bound to a ligand. In addition, although the sensors are easy to operate from a user perspective, purifying aTFs that are functional in vitro can be nontrivial. 
Freeze-dried ROSALIND sensors

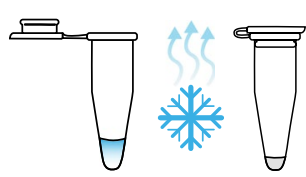

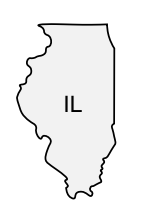

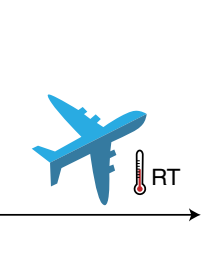

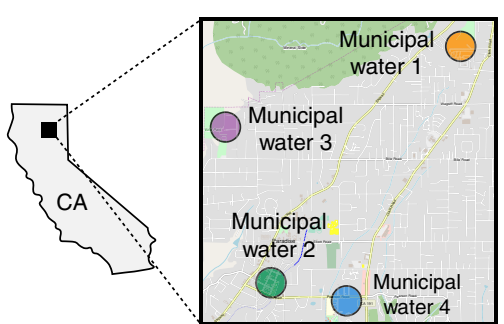

d
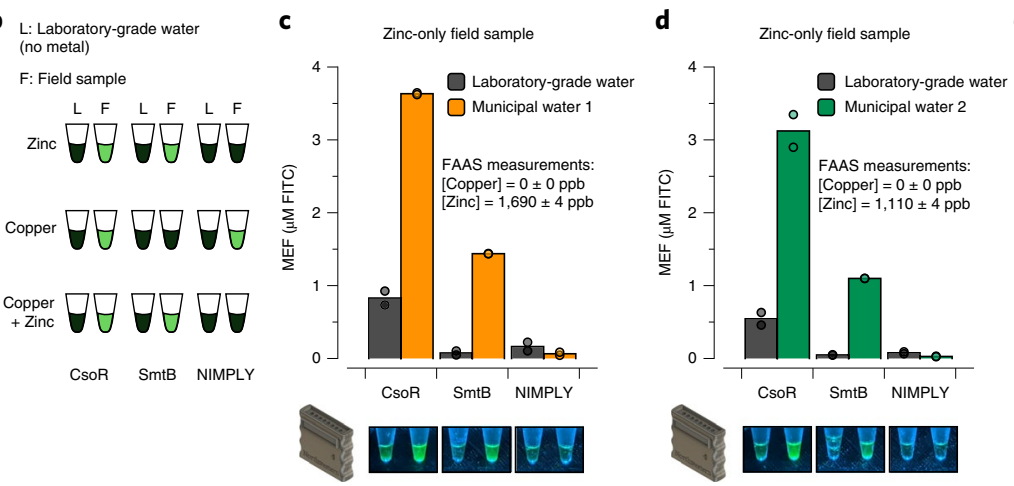

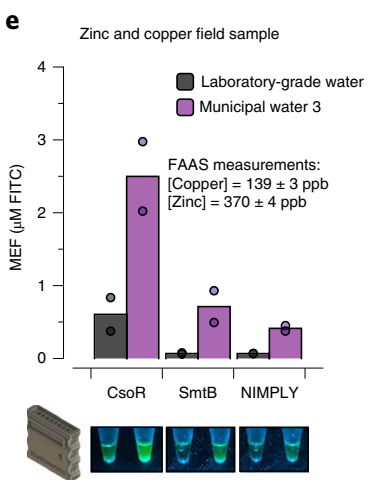

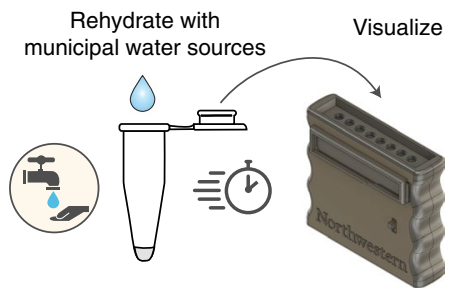

Fig. 6 | Field deployment of ROSALIND. a, ROSALIND reactions were freeze-dried in Evanston, IL, shipped at ambient conditions and applied to municipal water from Paradise, CA, where the water quality was affected by the destructive Camp Fire. Four different municipal water samples were collected, filtered and used to rehydrate the sensors, which were then incubated in a portable incubator. A 3D-printed, handheld illuminator was used to visualize signals from the rehydrated sensors within a day. $\mathbf{b}$, Three different types of sensors were shipped and tested in strips of tubes: zinc-sensing SmtB,

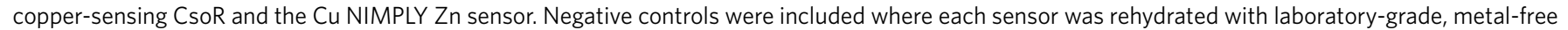
water. The expected results for different metal contaminations are shown. c-f, ROSALIND reactions rehydrated with Paradise municipal water samples from four locations, 1 (c), 2 (d), 3 (e) and 4 (f), were shipped back to Evanston for plate reader quantification (top), and aligned with handheld illuminator images (bottom). The collected field samples were tested with FAAS for metal concentration validation. All sensors shipped and tested resulted in expected signal patterns. Data from $n=2$ technical replicates are shown as points with raw fluorescence values standardized to MEF ( $\mu M$ FITC), and bars representing averages of two technical replicates. Images are shown for one replicate with other replicate images included in Supplementary Data 1 . The

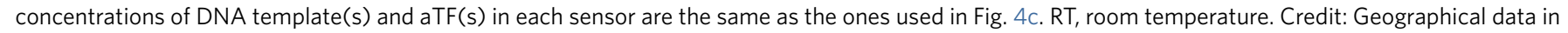
a (C) OpenStreetMap contributors ${ }^{46}$.

Finally, signal detection by eye can be difficult due to variations in lighting and reaction conditions. Although the inclusion of control reactions helps with this, a more sophisticated illuminator design that incorporates quantification would improve this aspect of the system.

A main feature of our study is demonstrating that freeze-dried ROSALIND reactions can be manufactured and shipped across the USA at ambient temperatures and applied to municipal water samples in the field (Fig. 6). We also found that ROSALIND reactions could be applied to other environmental water sources, although with some inhibition of the observed fluorescence levels (Extended Data Fig. 10). This can potentially be due to molecular components in the water samples that can inhibit transcriptional activity or quench fluorescence. Nevertheless, we did find that a practical workflow of testing serial dilutions could be used to detect copper, which we anticipate dilutes the interfering components in the reactions (Extended Data Fig. 10). In addition, different test formats may be used to include calibration curves that control for matrix effects ${ }^{50}$.

ROSALIND builds on significant earlier work in developing $\mathrm{WCBs}^{8}$ and cell extract-based biosensors ${ }^{2}$. Compared with WCBs, the defined in vitro environment of ROSALIND enables rapid optimization of sensor performance (Extended Data Fig. 2) and implementation of RNA-level circuitry to address key limitations of aTF-based sensors ${ }^{8}$. However, this flexibility comes at the cost of purifying functional aTFs and optimizing reaction conditions, and one advantage of WCBs is the simplicity of creating engineered strains. WCBs may take advantage of active transport to accumulate and detect only a bioavailable ligand, that is, the fraction that can cross the cell membrane ${ }^{9}$, although more systematic comparisons are needed to precisely define these benefits. Finally, WCBs require additional equipment or procedures to maintain cells in physiologically active states, encapsulation to manage biocontainment and normalization of signals to account for different numbers of cells in each reaction - challenges that are significantly mitigated by cell-free biosensing approaches such as ROSALIND.

Taken together, WCBs and cell-free synthetic biology approaches suchas ROSALIND help fillagapin existing water-quality-monitoring technology, which currently consists mainly of centralized and expensive instrumentation that cannot always be deployed in the field (Supplementary Table 4). We anticipate ROSALIND as part of a synthetic biology-based solution to enable communities and individuals to rapidly and economically monitor their water quality to meet their needs, to inform when and where more accurate measurements are needed and to facilitate the gathering of critical information necessary for achieving the United Nations Global Sustainable Development Goal of safe and clean water.

\section{Online content}

Any methods, additional references, Nature Research reporting summaries, source data, extended data, supplementary information, acknowledgements, peer review information; details of author contributions and competing interests; and statements of data and code availability are available at https://doi.org/10.1038/ s41587-020-0571-7. 
Received: 25 April 2019; Accepted: 19 May 2020;

Published online: 06 July 2020

\section{References}

1. French, K. E. Harnessing synthetic biology for sustainable development Nat. Sustain. 2, 250-252 (2019).

2. Slomovic, S., Pardee, K. \& Collins, J. J. Synthetic biology devices for in vitro and in vivo diagnostics. Proc. Natl Acad. Sci. USA 112, 14429-14435 (2015)

3. Pardee, $\mathrm{K}$. et al. Rapid, low-cost detection of Zika virus using programmable biomolecular components. Cell 165, 1255-1266 (2016).

4. Gootenberg, J. S. et al. Multiplexed and portable nucleic acid detection platform with Cas13, Cas12a, and Csm6. Science 360, 439-444 (2018).

5. Chen, J. S. et al. CRISPR-Cas12a target binding unleashes indiscriminate single-stranded DNase activity. Science 360, 436-439 (2018).

6. Takahashi, M. K. et al. A low-cost paper-based synthetic biology platform for analyzing gut microbiota and host biomarkers. Nat. Commun. 9, 3347 (2018).

7. Vorosmarty, C. J. et al. Global threats to human water security and river biodiversity. Nature 467, 555-561 (2010).

8. van der Meer, J. R. \& Belkin, S. Where microbiology meets microengineering: design and applications of reporter bacteria. Nat. Rev. Microbiol. 8, 511-522 (2010).

9. McNerney, M. P. et al. Active analyte import improves the dynamic range and sensitivity of a vitamin $\mathrm{B}_{12}$ biosensor. ACS Synth. Biol. 9, 402-411 (2020).

10. Belkin, $\mathrm{S}$. et al. Remote detection of buried landmines using a bacterial sensor. Nat. Biotechnol. 35, 308-310 (2017)

11. Siegal-Gaskins, D. et al. Gene circuit performance characterization and resource usage in a cell-free 'breadboard'. ACS Synth. Biol. 3, 416-425 (2014).

12. Alam, K. K. et al. A fluorescent split aptamer for visualizing RNA-RNA assembly in vivo. ACS Synth. Biol. 6, 1710-1721 (2017).

13. Zhang, Z. et al. Single-molecule tracking of the transcription cycle by sub-second RNA detection. eLife 3, e01775 (2014).

14. Pedelacq, J. D. et al. Engineering and characterization of a superfolder green fluorescent protein. Nat. Biotechnol. 24, 79-88 (2006).

15. Shimizu, Y. et al. Cell-free translation reconstituted with purified components. Nat. Biotechnol. 19, 751-755 (2001).

16. Cuthbertson, L. \& Nodwell, J. R. The TetR family of regulators. Microbiol. Mol. Biol. Rev. 77, 440-475 (2013).

17. Dunn, J. J. \& Studier, F. W. Complete nucleotide sequence of bacteriophage T7 DNA and the locations of T7 genetic elements. J. Mol. Biol. 166, 477-535 (1983).

18. Fernandez-Lopez, R. et al. Transcription factor-based biosensors enlightened by the analyte. Front. Microbiol. 6, 648 (2015).

19. Baquero, F., Martinez, J. L. \& Canton, R. Antibiotics and antibiotic resistance in water environments. Curr. Opin. Biotechnol. 19, 260-265 (2008).

20. Zhang, W. et al. Engineered biosynthesis of a novel amidated polyketide, using the malonamyl-specific initiation module from the oxytetracycline polyketide synthase. Appl. Environ. Microbiol. 72, 2573-2580 (2006).

21. Deochand, D. K. \& Grove, A. MarR family transcription factors: dynamic variations on a common scaffold. Crit. Rev. Biochem. Mol. Biol. 52, 595-613 (2017).

22. Zhu, T. et al. Deciphering and engineering of the final step halogenase for improved chlortetracycline biosynthesis in industrial Streptomyces aureofaciens. Metab. Eng. 19, 69-78 (2013)

23. Noguchi, N. et al. Regulation of transcription of the $\mathrm{mph}(\mathrm{A})$ gene for macrolide 2'-phosphotransferase I in Escherichia coli: characterization of the regulatory gene mphR(A). J. Bacteriol. 182, 5052-5058 (2000).

24. Richardson, S. D. \& Ternes, T. A. Water analysis: emerging contaminants and current issues. Anal. Chem. 90, 398-428 (2018).

25. Kasey, C. M. et al. Development of transcription factor-based designer macrolide biosensors for metabolic engineering and synthetic biology. ACS Synth. Biol. 7, 227-239 (2018).
26. Carballa, M. et al. Behavior of pharmaceuticals, cosmetics and hormones in a sewage treatment plant. Water Res. 38, 2918-2926 (2004).

27. Hiromoto, T. et al. Characterization of MobR, the 3-hydroxybenzoateresponsive transcriptional regulator for the 3-hydroxybenzoate hydroxylase gene of Comamonas testosteroni KH122-3s. J. Mol. Biol. 364, 863-877 (2006).

28. Kim, M. et al. Widely used benzalkonium chloride disinfectants can promote antibiotic resistance. Appl. Microbiol. 84, e01201-18. (2018).

29. Grkovic, S. et al. QacR is a repressor protein that regulates expression of the Staphylococcus aureus multidrug efflux pump QacA. J. Biol. Chem. 273, 18665-18673 (1998).

30. Lu, W. J. et al. Enantiomers of naringenin as pleiotropic, stereoselective inhibitors of cytochrome P450 isoforms. Chirality 23, 891-896 (2011).

31. Rogers, J. K. et al. Synthetic biosensors for precise gene control and real-time monitoring of metabolites. Nucleic Acids Res. 43, 7648-7660 (2015).

32. Liu, X. et al. De novo design of programmable inducible promoters. Nucleic Acids Res. 47, 10452-10463 (2019).

33. Juraschek, S. P. et al. Gout, urate-lowering therapy, and uric acid levels among adults in the United States. Arthrit. Care Res. 67, 588-592 (2015).

34. Wilkinson, S. \& Grove, A. HucR, a novel uric acid-responsive member of the MarR family of transcriptional regulators from Deinococcus radiodurans. J. Biol. Chem. 279, 51442-51450 (2004).

35. Abraham, J., Dowling, K. \& Florentine, S. Risk of post-fire metal mobilization into surface water resources: a review. Sci. Total Environ. 599, 1740-1755 (2017).

36. Hanna-Attisha, M. et al. Elevated blood lead levels in children associated with the Flint drinking water crisis: a spatial analysis of risk and public health response. Am. J. Public Health 106, 283-290 (2016).

37. Calle, G. R. et al. Enhanced copper release from pipes by alternating stagnation and flow events. Environ. Sci. Technol. 41, 7430-7436 (2007).

38. VanZile, M. L., Chen, X. \& Giedroc, D. P. Allosteric negative regulation of smt O/P binding of the zinc sensor, SmtB, by metal ions: a coupled equilibrium analysis. Biochemistry 41, 9776-9786 (2002).

39. Chang, F. M. et al. Cu(I)-mediated allosteric switching in a copper-sensing operon repressor (CsoR). J. Biol. Chem. 289, 19204-19217 (2014).

40. Busenlehner, L. S. et al. Spectroscopic properties of the metalloregulatory $\mathrm{Cd}(\mathrm{II})$ and $\mathrm{Pb}(\mathrm{II})$ sites of $S$. aureus $\mathrm{pI} 258 \mathrm{CadC}$. Biochemistry 40, 4426-4436 (2001)

41. Lloyd, J. et al. Dynamic control of aptamer-ligand activity using strand displacement reactions. ACS Synth. Biol. 7, 30-37 (2018).

42. Reyes-Caballero, H. et al. The metalloregulatory zinc site in Streptococcus pneumoniae AdcR, a zinc-activated MarR family repressor. J. Mol. Biol. 403 197-216 (2010)

43. Danner, M. C. et al. Antibiotic pollution in surface fresh waters: occurrence and effects. Sci. Total Environ. 664, 793-804 (2019).

44. Steber, M. et al. Mechanistic basis for RNA aptamer-based induction of TetR. ChemBioChem 12, 2608-2614 (2011).

45. Pardee, K. et al. Paper-based synthetic gene networks. Cell 159, 940-954 (2014).

46. Haklay, M. A. W. P. Openstreetmap: UserGenerated street maps. IEEE Pervas. Comput. 7, 12-18 (2008)

47. Vega, A. S., Lizama, K. \& Pastén, P. A. in Water Policy in Chile (ed. Donoso, G.) 25-51 (Springer International Publishing, 2018).

48. Chen, X. et al. Visualizing RNA dynamics in live cells with bright and stable fluorescent RNAs. Nat. Biotechnol. 37, 1287-1293 (2019).

49. Ellefson, J. W., Ledbetter, M. P. \& Ellington, A. D. Directed evolution of a synthetic phylogeny of programmable Trp repressors. Nat. Chem. Biol. 14, 361-367 (2018).

50. McNerney, M. P. et al. Point-of-care biomarker quantification enabled by sample-specific calibration. Sci. Adv. 5, eaax4473 (2019).

Publisher's note Springer Nature remains neutral with regard to jurisdictional claims in published maps and institutional affiliations.

(c) The Author(s), under exclusive licence to Springer Nature America, Inc. 2020 


\section{Methods}

Strains and growth medium. Escherichia coli strain K12 (NEB Turbo Competent E. coli, New England Biolabs, catalog no. C2984) was used for routine cloning. E. coli strain Rosetta 2(DE3)pLysS (Novagen, catalog no. 71401) was used for recombinant protein expression. Lysogeny broth supplemented with the appropriate antibiotic(s) $\left(100 \mu \mathrm{g} \mathrm{ml}^{-1}\right.$ carbenicillin, $100 \mu \mathrm{g} \mathrm{ml}^{-1} \mathrm{kanamycin}$ and/or $34 \mu \mathrm{g} \mathrm{ml}^{-1}$ chloramphenicol) was used as the growth medium.

Plasmids and genetic parts assembly. DNA oligonucleotides for cloning and sequencing were synthesized by Integrated DNA Technologies. Genes encoding aTFs were synthesized either as gBlocks (Integrated DNA Technologies) or gene fragments (Twist Bioscience). Expression plasmids were cloned using Gibson Assembly (NEB Gibson Assembly Master Mix, New England Biolabs, catalog no. E2611) into a pET$28 \mathrm{c}$ plasmid backbone and were designed to overexpress recombinant proteins as either $\mathrm{C}$ terminus or $\mathrm{N}$ terminus, His-tagged fusions. Certain constructs additionally incorporated a recognition sequence for cleavage and removal of the His-tag using tobacco etch virus protease (Supplementary Table 5). Gibson-assembled constructs were transformed into NEB Turbo cells, and isolated colonies were purified for plasmid DNA (QIAprep Spin Miniprep Kit, Qiagen, catalog no. 27106). Plasmid sequences were verified with Sanger DNA sequencing (Quintara Biosciences) using the primers listed in Supplementary Data 5.

Plasmids encoding transcription templates were constructed using pUC19-T73WJdB-T (Addgene, catalog no. 87308) as a backbone. These plasmids included a T7 RNAP promoter, an aTF operator site, the $3 \mathrm{WJdB}$ coding sequence ${ }^{12}$ and a T7 terminator. In the present study, we define the T7 RNAP promoter as a minimal 17-bp sequence (TAATACGACTCACTATA) excluding the first G that is transcribed. Plasmids were cloned using either Gibson Assembly or inverse PCR followed by blunt-end ligation, and then transformed into NEB Turbo cells for clonal isolation and sequence verification using the primers listed in Supplementary Data 5. Once the sequence had been verified, transcription templates were generated by PCR amplification (Phusion High-Fidelity PCR Kit, New England Biolabs, catalog no. E0553) of the plasmids to include a $5^{\prime}$-region upstream of the T7 promoter and a $3^{\prime}$-region ending with either $3 \mathrm{WJdB}$ or the T7 terminator, with the primers listed in Supplementary Data 5. Amplified templates were then purified (QIAquick PCR purification kit, Qiagen, catalog no. 28106) and verified for the presence of a single DNA band of expected size on a $2 \%$ Trisacetate-EDTA (TAE)-agarose gel, and concentrations were determined using the Qubit dsDNA BR Assay Kit (Invitrogen, catalog no. Q32853).

The KB2 DNA transcription templates were generated by denaturing two complementary oligonucleotides (Supplementary Data 5) at $95^{\circ} \mathrm{C}$ for $3 \mathrm{~min}$ and slow cooling $\left(-0.1^{\circ} \mathrm{C} \mathrm{s}^{-1}\right)$ to room temperature in $1 \times$ annealing buffer $(100 \mathrm{mM}$ potassium acetate and $30 \mathrm{mM}$ HEPES, $\mathrm{pH}$ 8.0). Annealed oligonucleotides were then purified by resolving them on a $20 \%$ Tris-borate-EDTA (TBE)-PAGE gel, isolating the band of expected size and eluting at room temperature overnight in $1 \times$ annealing buffer. The eluted DNA template was then ethanol precipitated, resuspended in MilliQ $\mathrm{H}_{2} \mathrm{O}$ and quantified using the Qubit dsDNA BR Assay Kit. The KB2 DNA template for the Cu NIMPLY Zn circuit was constructed by placing the smtO sequence 5-bp downstream of the T7 promoter and immediately upstream of the KB2-coding sequence ${ }^{41}$. The NIMPLY control circuit was designed by reversing the KB2 sequence, while keeping the other parts consistent. These DNA templates were generated using the same method.

The anti-TetR DNA-transcription template was constructed by placing the tet $O$ sequence $4 \mathrm{bp}$ downstream of the $\mathrm{T} 7$ promoter and immediately upstream of the anti-TetR-coding sequence ${ }^{44}$. This template was generated by three sequential PCR amplifications using primers listed in Supplementary Data 5. The first PCR reaction was performed using a single-stranded DNA oligonucleotide as its template, and the resulting PCR product was used as the template for the second PCR reaction. After the second PCR amplification, the expected DNA band was extracted from a 2\% agarose gel (QIAquick Gel Extraction Kit, Qiagen, catalog no. 28706) for the final PCR amplification. Amplified templates were then purified and verified for the presence of a single DNA band of expected size on a $2 \%$ TAE-agarose gel, and concentrations were determined using the Qubit dsDNA BR Assay Kit. The anti-TetR control circuit was designed by reversing the anti-TetR sequence, and its DNA template was generated using the same method.

The sequences and Addgene accession numbers of all plasmids generated in the present study are listed in Supplementary Data 5.

Expression and purification of aTFs. Supplementary Table 5 provides a listing of the exact expression and purification process for each aTF. In general, with the exception of CadC and AdcR, each protein was expressed and purified as follows: sequence-verified pET-28c plasmids were transformed into the Rosetta 2(DE3) pLysS E. coli strain for protein expression. A single transformed colony from each expression plasmid transformation was then used to seed a small overnight culture grown at $37^{\circ} \mathrm{C}$. Overnight cultures were then seeded into larger expression cultures ( $10 \mathrm{ml}$ of starter culture per 11 of expression culture). Cultures were induced for overexpression at an optical density $(600 \mathrm{~nm})$ of $\sim 0.5$ with $100-500 \mu \mathrm{M}$ isopropyl $\beta$-D-1-thiogalactopyranoside and grown for $4 \mathrm{~h}$ more at $37^{\circ} \mathrm{C}$ or overnight at $30^{\circ} \mathrm{C}$. Cultures were then pelleted by centrifuging at $4,000 \mathrm{~g}$ for $20 \mathrm{~min}$ at $4{ }^{\circ} \mathrm{C}$ in a Thermo Scientific Sorvall Lynx 4000, and then were either immediately stored at $-80^{\circ} \mathrm{C}$ or resuspended in lysis buffer $(10 \mathrm{mM}$ Tris- $\mathrm{HCl}, \mathrm{pH} 7.5-8.5$, $500 \mathrm{mM} \mathrm{NaCl}, 1 \mathrm{mM}$ tris(2-carboxyethyl)phosphine (TCEP) and protease inhibitor (cOmplete EDTA-free Protease Inhibitor Cocktail, Roche)). Resuspended cells were then lysed on ice through four rounds of ultrasonication ( $50 \%$ duty cycle) for $1 \mathrm{~min}$, with $2 \mathrm{~min}$ of rest on ice in between rounds. Lysates were then centrifuged at $13,000 \mathrm{~g}$ for $30 \mathrm{~min}$ to remove insoluble material. Clarified supernatants were purified using His-tag affinity chromatography with an Ni-NTA column (HisTrap FF 5-ml column, GE Healthcare Life Sciences), followed by size exclusion chromatography (Superdex HiLoad 26/600 200-pg column, GE Healthcare Life Sciences) using an AKTAxpress fast protein liquid chromatography system. The collected fractions from the fast protein liquid chromatography were concentrated and buffer exchanged ( $25 \mathrm{mM}$ Tris- $\mathrm{HCl}, 100 \mathrm{mM} \mathrm{NaCl}, 1 \mathrm{mM}$ TCEP, 50\% glycerol, v/v) using centrifugal filtration (Amicon Ultra-0.5, Millipore Sigma). Protein concentrations were determined using the Qubit Protein Assay Kit (Invitrogen, catalog no. Q33212). The purity and size of the proteins were validated on SDSPAGE gel (Mini-PROTEAN TGX and Mini-TETRA cell, Bio-Rad). Purified proteins were stored at $-20^{\circ} \mathrm{C}$. CadC and $\mathrm{AdcR}$, the genes of which were cloned into pET-3d and pET-3a plasmid backbones, respectively, were purified with the protocol previously described ${ }^{51}$. For CadC purification, the protocol was modified to have a higher concentration of TCEP $(5 \mathrm{mM})$ in its buffers.

IVT reactions. NEB HiScribe T7 Quick High Yield RNA Synthesis Kit (New England Biolabs, catalog no. E2050S) was used for the data shown in Fig. 2b,d, Supplementary Figs. 2 and 3, and Extended Data Fig. 1, according to the manufacturer's protocol, with the addition of $2.25 \mathrm{mM}$ DFHBI-1T (Tocris, catalog no. 5610) in the reaction mix. Homemade IVT reactions were set up by adding the following components listed at their final concentration: IVT buffer ( $40 \mathrm{mM}$ Tris- $\mathrm{HCl}$, pH 8, $8 \mathrm{mM} \mathrm{MgCl}_{2}, 10 \mathrm{mM}$ DTT, $20 \mathrm{mM} \mathrm{NaCl}$ and $2 \mathrm{mM}$ spermidine), 2.25 mM DFHBI-1T, 11.4 mM Tris-buffered nucleotide triphosphates, $\mathrm{pH} 7.5$, $0.3 \mathrm{U}$ thermostable inorganic pyrophosphatase (New England Biolabs, catalog no. M0296S), DNA transcription template(s) and MilliQ $\mathrm{H}_{2} \mathrm{O}$ to a total volume of $20 \mu \mathrm{l}$. Regulated IVT reactions additionally included a purified aTF at the indicated concentration and were equilibrated at $37^{\circ} \mathrm{C}$ for $15 \mathrm{~min}$. Immediately before plate reader measurements, $0.2 \mathrm{ng}$ of T7 RNAP and, optionally, a ligand at the indicated concentration (Supplementary Table 2) were added to the reaction. Reactions were then characterized on a plate reader as described under Plate reader quantification and MEF standardization. Supplementary Data 2 contains a master experimental planning sheet that describes how a typical ROSALIND reaction is set up.

NEB PURExpress In Vitro Protein Synthesis Kit (New England Biolabs, catalog no. E6800) was used for the data shown in Fig. 2d and Supplementary Fig. 3, according to the manufacturer's protocol. Linear DNA template encoding a T7 promoter, followed by sfGFP and a T7 terminator, was PCR amplified (Phusion High-Fidelity PCR Kit, New England Biolabs, catalog no. E0553) from the plasmid pJL1 (Addgene, catalog no. 69496) using primers listed in Supplementary Data 5. Amplified template was then purified, quantified using the Qubit dsDNA BR Assay Kit and verified for the presence of a single band of expected size on a $1 \%$ TAE-agarose gel.

Freeze-drying. Before lyophilization, PCR tube caps were punctured with a pin to create three holes. Lyophilization of ROSALIND reactions was then performed by assembling the components of regulated IVT (see IVT reactions) with the addition of $50 \mathrm{mM}$ sucrose and $250 \mathrm{mM} \mathrm{D}$-mannitol. Assembled reaction tubes were immediately transferred into a prechilled aluminum block and placed in a $-80^{\circ} \mathrm{C}$ freezer for $10 \mathrm{~min}$ to allow slow freezing. After the slow freezing, reaction tubes were wrapped in Kimwipes and aluminum foil, submerged in liquid nitrogen and then transferred to a FreeZone 2.5-1 Bench Top Freeze Dry System (Labconco) for overnight freeze-drying with a condenser temperature of $-85^{\circ} \mathrm{C}$ and $0.04 \mathrm{mbar}$ of pressure. Unless rehydrated immediately, freeze-dried reactions were packaged as follows: the reactions were placed in a light-protective bag (Mylar open-ended food bags, Uline, catalog no. S-11661) with a desiccant (Dri-Card Desiccants, Uline, catalog no. S-19582), purged with argon using an argon canister (ArT Wine Preserver, Amazon, Amazon Standard Identification Number (ASIN) B01MEHJCQ2) and immediately impulse heat sealed (Metronic 8 inch Impulse Bag Sealer, Amazon, ASIN B06XC76JVZ) (Supplementary Video 2).

Plate reader quantification and MEF standardization. A National Institute of Standards and Technology (NIST) traceable standard (Invitrogen, catalog no. F36915) was used to convert arbitrary fluorescence measurements to MEF. Serial dilutions from a $50-\mu \mathrm{M}$ stock were prepared in $100 \mathrm{mM}$ sodium borate buffer at $\mathrm{pH} 9.5$, including a 100-mM sodium borate buffer blank (total of 12 samples). The samples were prepared in technical and experimental triplicates (12 samples $\times 9$ replicates $=108$ samples total), and fluorescence values were read at an excitation wavelength of $472 \mathrm{~nm}$ and emission wavelength of $507 \mathrm{~nm}$ for 3WJdB-activated fluorescence, or 485-nm excitation and 515-nm emission for sfGFP fluorescence on a plate reader (Synergy H1, BioTek). Fluorescence values for a concentration in which a single replicate saturated the plate reader were excluded from analysis. The remaining replicates (nine per sample) were then averaged at each FITC concentration, and the average fluorescence value of the blank was subtracted from all values. Linear regression was then performed for 
concentrations within the linear range of fluorescence $(0-6.25 \mu \mathrm{M}$ FITC) between the measured fluorescence values in arbitrary units and the concentration of FITC for identifying the conversion factor. For each plate reader, excitation, emission and gain setting, we found a linear conversion factor that was used to correlate arbitrary fluorescence values to MEF ( $\mu$ M FITC) (Supplementary Data 3).

To characterize ROSALIND reactions, $19-\mu$ ROSALIND reactions were loaded onto a 384-well, black, optically clear, flat-bottomed plate using a multichannel pipette and measured on a plate reader (Synergy H1, BioTek). Kinetic analysis was performed by reading the plate at 3-min intervals with excitation and emission wavelengths of 472 and $507 \mathrm{~nm}$, respectively, for $4 \mathrm{~h}$ at $37^{\circ} \mathrm{C}$. PURExpress reactions were quantified and standardized to MEF ( $\mu \mathrm{M}$ FITC) as described for ROSALIND reactions, but with excitation and emission wavelengths set to 485 and $515 \mathrm{~nm}$, respectively.

We note that no background subtraction was performed when analyzing outputs from any ROSALIND reaction. An example of this standardization procedure is shown in Supplementary Fig. 1.

Municipal and surface water sampling. Municipal water was collected from water fountains in public parks of Paradise, CA, in October of 2019, as the town recovered from the most destructive wildfire in California's history (Camp Fire, November 2018). This urban fire severely damaged the municipal water system in Paradise, resulting in widespread contamination of drinking water pipes. After the fire, a Water Quality Advisory was issued, asking the public not to drink municipal water. With the Water Quality Advisory still in effect at the time of sampling, public water fountains had probably remained unflushed for $\sim 11$ months. Water samples were collected from self-standing water spigots (brass or stainless steel) in metal-free plastic tubes and filtered $(0.45-\mu$ m nylon membranes $)$ in the field using acid-washed plastic syringes. Then, 50 -ml filtered subsamples were immediately acidified to $\mathrm{pH} \sim 2$ with $\mathrm{HNO}_{3}$ for metal analysis (FAAS) and kept cold $\left(4^{\circ} \mathrm{C}\right.$ ) with 50-ml filtered, unacidified subsamples until their return to California State University, Chico, where ROSALIND tests were initiated within $4 \mathrm{~h}$ of collection using filtered, unacidified subsamples.

Tests were initiated by applying $20 \mu \mathrm{l}$ of filtered unacidified samples to reaction tubes, or laboratory-grade, metal-free water (ultrapure type 1 water, resistivity $>18 \mathrm{M} \Omega \mathrm{cm}$ ), as indicated. Reactions were incubated in a portable incubator at $37^{\circ} \mathrm{C}$ and periodically monitored. Photographs were taken of reaction tubes after $2 \mathrm{~h}$ and overnight using the handheld illuminator.

Reaction strips and water samples were shipped back to Northwestern University for fluorescence measurements by the plate reader (see Plate reader quantification and MEF standardization) and metal analysis (see FAAS measurement).

For Chilean environmental samples, river water samples were taken from the Yerba Loca Creek in the upper Mapocho-Maipo watershed, and the Toro River in the upper Elqui River watersheds. Both streams are metal-polluted tributaries to rivers that provide ecosystem services (for example, drinking water sources, habitat for ecosystems and recreation) to major Chilean cities. Acid-washed high-density polypropylene bottles were used to collect $200 \mathrm{ml}$ of filtered $(0.45-\mu \mathrm{m}$ nylon membranes) samples and were shipped in a cooler. Given the low $\mathrm{pH}$ $(2.9<\mathrm{pH}<4)$ and the objective of the experiments, the $\mathrm{pH}$ was not adjusted.

For copper/zinc-spiked municipal water from Evanston, IL, two bottles of approximately $250 \mathrm{ml}$ of the water samples were collected from a drinking fountain. One of the bottles was then filtered at $0.45 \mu \mathrm{m}$ using $47-\mathrm{mm}$ cellulose acetate filter membranes (Thermo Fisher Scientific). The other was spiked with a known amount of $\mathrm{CuSO}_{4} / \mathrm{ZnSO}_{4}$ and then filtered using the same method. Then, the copper/zinc-spiked fountain water was diluted with the filtered nonspiked fountain water to create $40-\mathrm{ml}$ solutions in the range of $0.125-4 \mathrm{ppm}$ for copper and $0.05-3 \mathrm{ppm}$ for zinc. Of these water samples, $10 \mathrm{ml}$ was then acidified with $\mathrm{HNO}_{3}$ and measured using FAAS for concentration validation (see FAAS measurement) Of the water samples, $20 \mu \mathrm{l}$ was used to rehydrate freeze-dried CsoR, SmtB and $\mathrm{Cu}$ NIMPLY Zn sensors. Upon rehydration, fluorescence measurements of the reactions were performed by a plate reader (see "Plate reader quantification and MEF standardization"). For the copper-spiked Michigan water sample, the same method was used as for the Lake Michigan water sample.

FAAS measurement. An FAAS instrument (PerkinElmer PinAAcle 500) equipped with PerkinElmer Lumina hollow-cathode lamps was used to determine copper and zinc concentrations in the drinking water spiked with copper and zinc (Supplementary Fig. 6), the lake water spiked with copper, the Chilean surface water samples (Extended Data Fig. 10) and the Paradise municipal water samples (Fig. 6 and Supplementary Fig. 7). The spectral lines were $324.8 \mathrm{~nm}$ for copper and $213.9 \mathrm{~nm}$ for zinc. FAAS was calibrated for $\mathrm{Cu}$ (II) using $\mathrm{CuNO}_{3}$ reference solution as a standard (Thermo Fisher Scientific, catalog no. CAS10031-43-3) and for Zn(II) using certified International Organization for Standardization Guide 17034 standard solution (Fluka Analytical, catalog no. 18562), with calibration curves comprising between 0.05 and $2 \mathrm{ppm}$ for copper and 0.05 and $1 \mathrm{ppm}$ for zinc. Samples were diluted with $18 \mathrm{M} \Omega \mathrm{cm}$ deionized water (Millipore), when necessary, and acidified with ultrapure $\mathrm{HNO}_{3}$ (Fisher Chemical, catalog no. CAS7697-37-2). Three replicates were performed and averaged, and error propagations were taken into account to estimate uncertainties.
Photographs of reactions. Photographs of reaction tubes in Figs. 2 and 6 were taken on an Apple iPhone X and a Google Pixel 3a using their default camera applications, respectively. Photographs in Extended Data Fig. 8 were taken on an Apple iPhone 6S using the default Apple iOS Camera application. The resulting images were only cropped. Source image files are available as Supplementary Data 1.

Statistics and reproducibility. The number of replicates and types of replicates performed are described in the legend to each figure. Individual data points are shown and, where relevant, the mean \pm s.d.; this information is provided for each figure. The type of statistical analysis performed in Fig. 5 and Extended Data Fig. 4 is described in the legend to each figure. Exact $P$ values, along with degrees of freedom computed from the statistical analysis, can be found in Supplementary Data 3.

Reporting Summary. Further information on research design is available in the Nature Research Reporting Summary linked to this article.

\section{Data availability}

All data presented in this manuscript are available as supplementary data files. All plasmids used in this manuscript are available in Addgene with the identifiers 140371-140399. Source data are provided with this paper.

\section{References}

51. Capdevila, D. A. et al. Tuning site-specific dynamics to drive allosteric activation in a pneumococcal zinc uptake regulator. eLife 7, e37268 (2018).

\section{Acknowledgements}

We thank A. Thompson (Northwestern University) for managing the experimental reagents and equipment used in this study; Z. Yang (Northwestern University) for assistance with FAAS measurements; N. Donghia (Wyss Institute) for helpful discussions on lyophilization of cell-free reactions; S. Pshenychny (Recombinant Protein Production Core at Northwestern University) for assistance in protein purification; J. Webster, R. Gruenberg, J. Machado and E. Plasencia-Campos (CSU, Chico) for assisting with field work; and J. Bussan, F. Lantz and B. Golenia (Northwestern University Research Shop) for assistance in the development of the handheld illuminator. J.K.J. and M.S.V. were supported in part by Northwestern University's Graduate School Cluster in Biotechnology, System, and Synthetic Biology, which is affiliated with the Biotechnology Training Program. P.R.C. was supported in part by the National Science Foundation (NSF) Synthetic Biology REU (grant no. DBI-1757973). This work was also supported by funding from the Pew Charitable Trusts (to D.A.C.), the National Institutes of Health (grant no. R35 GM118157 to D.P.G.), CONICYT/FONDECYT (grant no. 1161337 to P.A.P.), ANID/FONDAP (grant no. 15110020 to P.A.P.), NSF CAREER (grant no. 1452441 to J.B.L.), NSF MCB RAPID (grant no. 1929912 to J.B.L., J.-F.G. and S.J.M.), support from the Crown Family Center for Jewish and Israel Studies at Northwestern University (to J.B.L.) and Searle Funds at the Chicago Community Trust (to J.B.L.).

\section{Author contributions}

J.K.J., K.K.A., J.J.C. and J.B.L. conceived the project. J.K.J., K.K.A., M.S.V. and J.B.L. curated the data. J.K.J., K.K.A. and J.B.L. carried out the formal analysis. J.B.L., J.-F.G., S.J.M., D.P.G. and P.A.P. acquired the funds. J.K.J., K.K.A., M.S.V., M.D., P.R.C., J.W.L. and J.-F.G. carried out the investigations. J.K.J., K.K.A., M.S.V., J.W.L., P.Q.N., D.A.C., M.D., S.J.M., J.-F.G. and J.B.L. devised the methodology. J.K.J., K.K.A. and J.B.L. administered the project. D.A.C., D.P.G., P.A.P. and S.J.M. acquired the resources. J.K.J., K.K.A., J.J.C. and J.B.L. supervised the project. J.K.J., K.K.A., M.S.V., M.D., P.R.C. and J.-F.G. validated the project. J.K.J., K.K.A. and J.B.L. visualized the project. J.K.J., K.K.A. and J.B.L. wrote the original draft. J.K.J., K.K.A., M.S.V., M.D., P.R.C., J.W.L., P.Q.N., D.A.C., P.A.P., S.J.M., J.-F.G., D.P.G., J.J.C. and J.B.L. reviewed and edited the manuscript.

\section{Competing interests}

K.K.A., J.K.J. and J.B.L. have submitted a US provisional patent application (no. $62 / 758,242$ ) relating to regulated IVT reactions. K.K.A., J.K.J., M.S.V., P.R.C., J.W.L., J.J.C. and J.B.L. have submitted a US provisional patent application (no. 62/838,852) relating to the preservation and stabilization of IVT reactions. K.K.A. and J.B.L. are founders and have a financial interest in Stemloop, Inc. The latter interests are reviewed and managed by Northwestern University in accordance with their conflict-of-interest policies. All other authors declare no conflicts of interest.

\section{Additional information}

Extended data is available for this paper at https://doi.org/10.1038/s41587-020-0571-7. Supplementary information is available for this paper at https://doi.org/10.1038/ s41587-020-0571-7.

Correspondence and requests for materials should be addressed to J.B.L.

Reprints and permissions information is available at www.nature.com/reprints. 


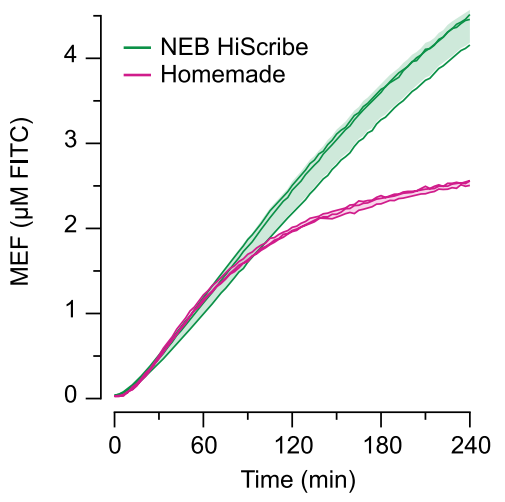

Extended Data Fig. 1 | Homemade transcription reactions can be optimized to perform as well as commercial kits. Homemade in vitro transcription reactions were compared to a commercially available high yield transcription kit (NEB HiScribe ${ }^{\mathrm{TM}}$ T7 Quick High Yield RNA Synthesis Kit). $25 \mathrm{nM}$ DNA encoding T7-3WJdB-T was added to each reaction in a total reaction volume of $20 \mu \mathrm{L}$. Over the course of 1 hour, the data show similar fluorescence activation. However, the homemade reaction begins to saturate after 1 hour, likely due to the exhaustion of NTPs in the homemade reaction (11.4 mM) when compared to the commercial kit ( $40 \mathrm{mM}$ ). All data shown for $n=3$ independent biological replicates as lines with raw fluorescence values standardized to MEF ( $\mu$ M FITC). Shading indicates the average value of 3 independent biological replicates \pm s.d. 


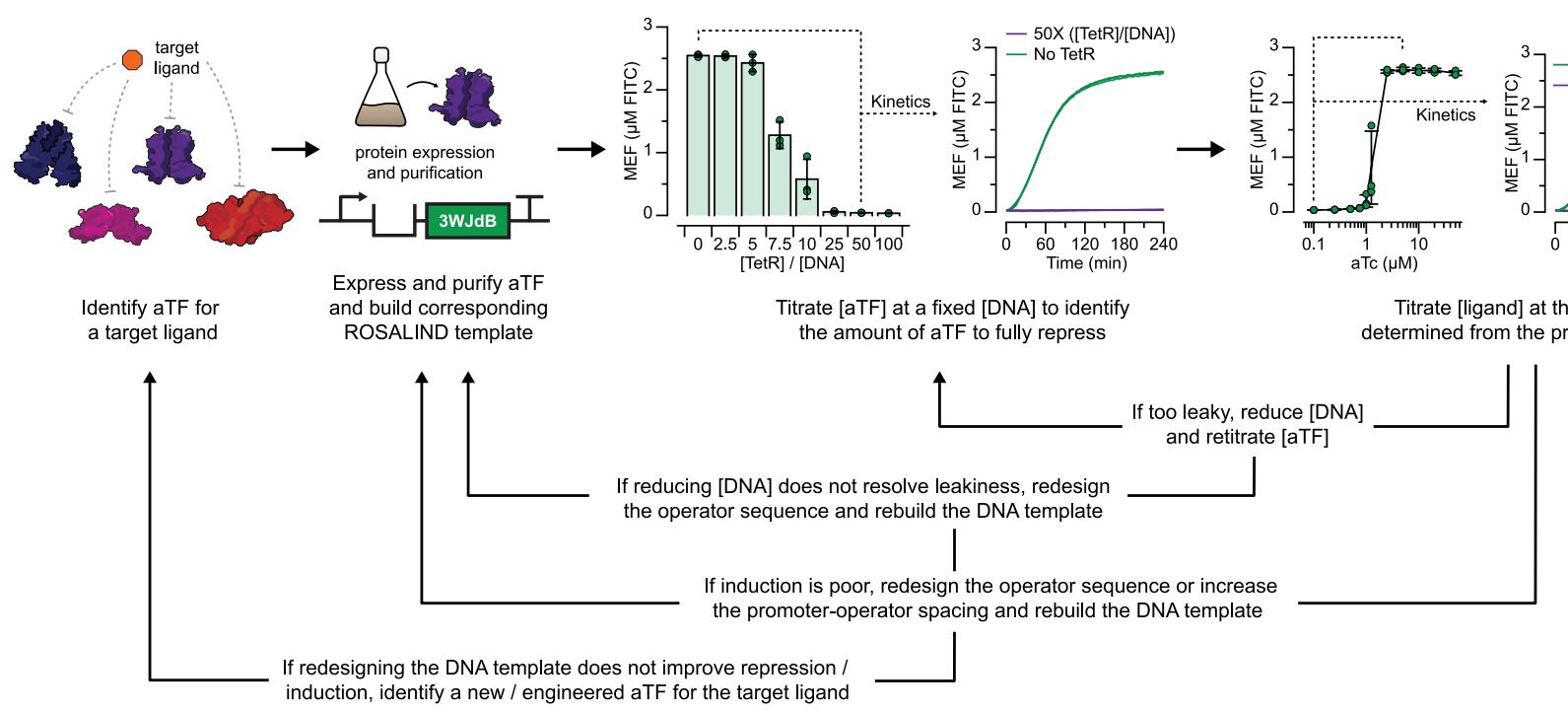

Extended Data Fig. 2 | Design and optimization of a ROSALIND reaction. Constructing a ROSALIND reaction begins with identifying a ligand of interest, an aTF capable of binding or unbinding a DNA operator sequence as a function of the ligand and the aTF's cognate operator sequence. Once identified, two DNA constructs are designed, one to separately express and purify the aTF and the other to generate a linear transcription template encoding the promoter, spacer, operator, fluorescent RNA aptamer and optional terminator. The first step in optimizing the sensor is performing a titration of the purified aTF at a fixed DNA template concentration to determine the amount of aTF needed to fully repress the expression of $3 \mathrm{WJdB}$. Then, the target ligand is titrated (at the concentration of aTF previously determined) to test for induction. If the sensor needs improvement, components of ROSALIND can be redesigned and retested. The data shown here are for the TetR-based ROSALIND reaction described in Fig. 2f,h. The bar graphs shown are data taken at $4 \mathrm{~h}$ after initiating reactions with T7 RNAP and $25 \mathrm{nM}$ DNA template, and the kinetics data are shown for $0 \mu \mathrm{M}$ (green lines) and $1.25 \mu \mathrm{M}$ TetR dimer (purple lines). Fluorescence-activation is substantially repressed at 25-fold excess or greater of TetR dimer over DNA template. The TetR-aTc dose response curve shown are the data presented in Fig. $2 \mathrm{~h}$, and the kinetics data are shown for $0 \mu \mathrm{M}$ (purple lines) and $2.5 \mu \mathrm{M}$ aTc (green lines). All data shown for $n=3$ independent biological replicates as points or lines with raw fluorescence values standardized to MEF ( $\mu M$ FITC), and bars representing averages of the replicates. Shading and error bars indicate the average value of 3 independent biological replicates \pm s.d. 
a

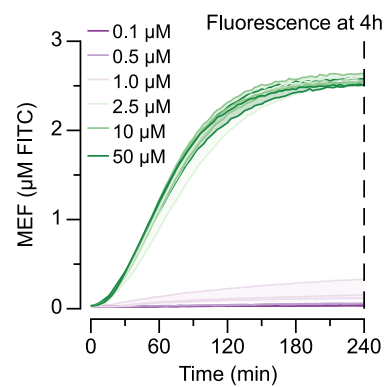

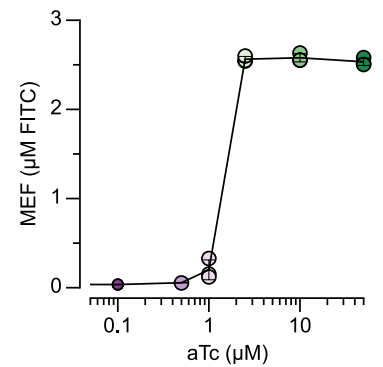

Extended Data Fig. 3 | Characterization of a ROSALIND dose response curve. A dose response curve of a TetR-regulated ROSALIND reaction is plotted from the measured and calibrated kinetics traces, using the 4-h end point fluorescence values at different ligand concentrations. $\mathbf{a}$, kinetic traces of TetR-aTc induction at different aTc concentrations, and $\mathbf{b}$, the corresponding 4-h fluorescence values plotted against the $\mu \mathrm{M}$ of aTc added. Reactions are generated using $25 \mathrm{nM}$ of DNA and $1.25 \mu \mathrm{M}$ of TetR dimer. All data shown for $\mathrm{n}=3$ independent biological replicates as lines (a) or points (b) with raw fluorescence values standardized to MEF ( $\mu$ M FITC). Shading (a) and error bars (b) indicate the average value of 3 independent biological replicates \pm s.d. 
a

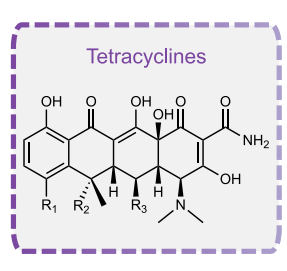

b

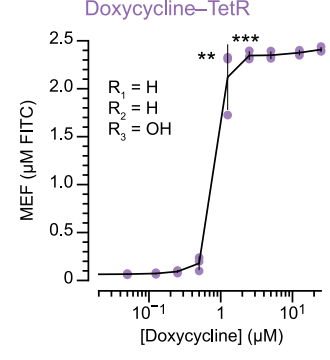

f
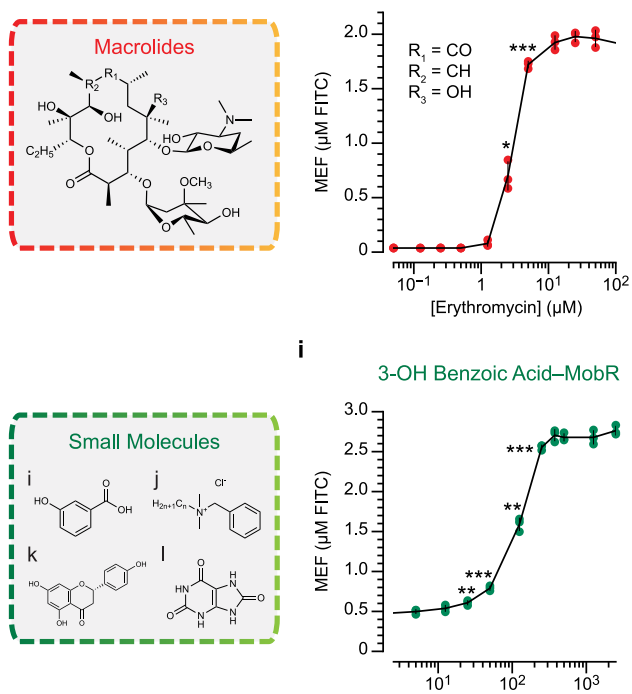

[3-OH Benzoic Acid] $(\mu \mathrm{M})$

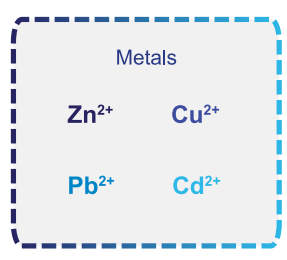

m

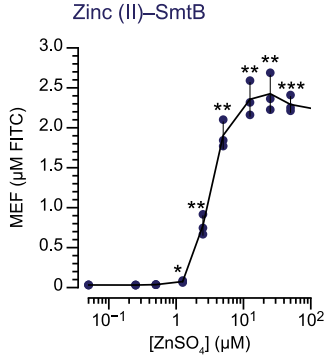

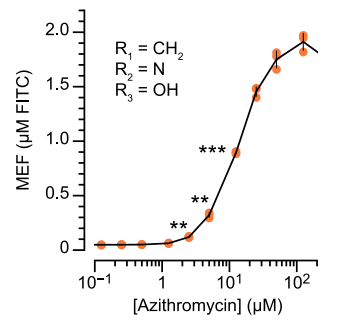

j

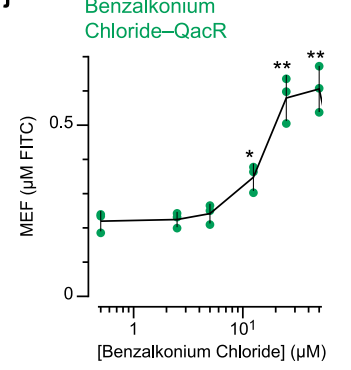

n

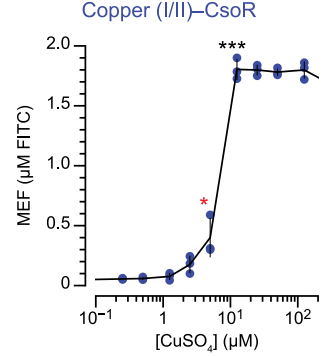

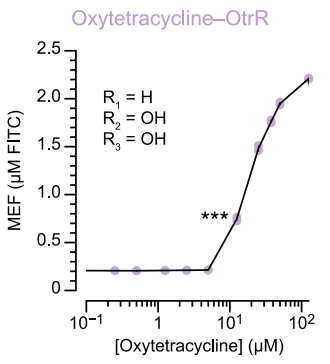

g

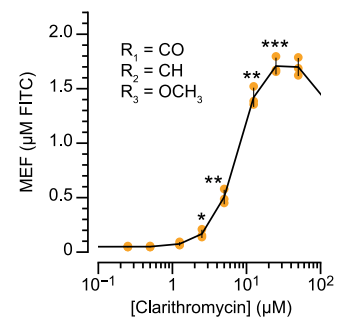

k

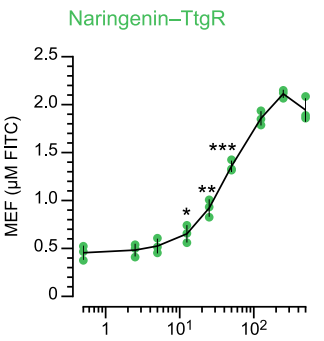

o

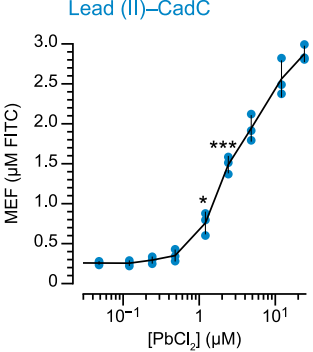

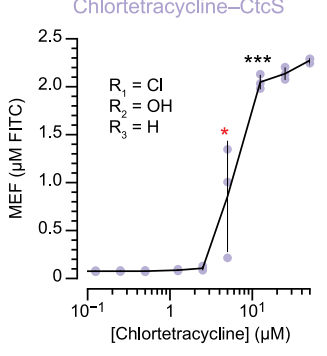

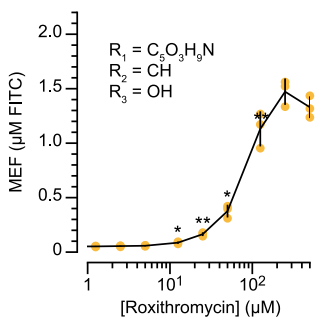

I

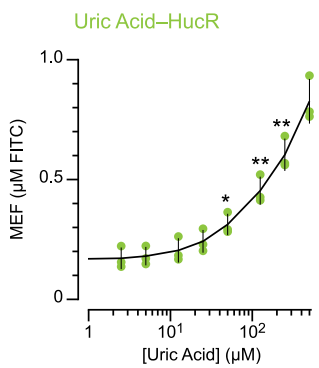

p

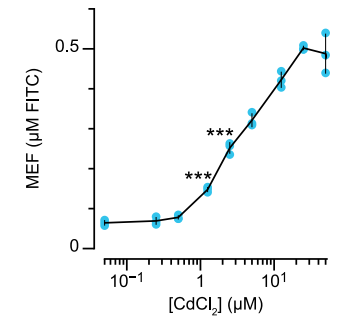

Extended Data Fig. 4 | Dose response curves of ROSALIND reactions. The dose response curves of ROSALIND reactions shown in Fig. 3 are presented. The amount of DNA templates and aTF used in each panel are configured as described in Supplementary Table 2. All data shown for $n=3$ independent biological replicates as points with raw fluorescence values standardized to MEF ( $\mu$ M FITC). Error bars indicate the average value of 3 independent biological replicates \pm s.d. The ligand concentrations at which the signal is distinguishable from the background were determined using two-tailed heteroscedastic Student's $t$-test against the no ligand condition, and their p-value ranges are indicated with black asterisks ${ }^{* \star *}<0.001,{ }^{\star \star}=0.001-0.01$, ${ }^{*}=0.01-0.05$ ). The asterisks indicated in red have $p$-values in the range between $0.05-0.15$ due to variability between replicates, although the average signals were clearly above background. Exact $p$-values along with degrees of freedom can be found in Supplementary Data 3. Data for no ligand conditions were excluded in the plots since the $x$-axis is on the log scale, but they can be found in Supplementary Data 3. 


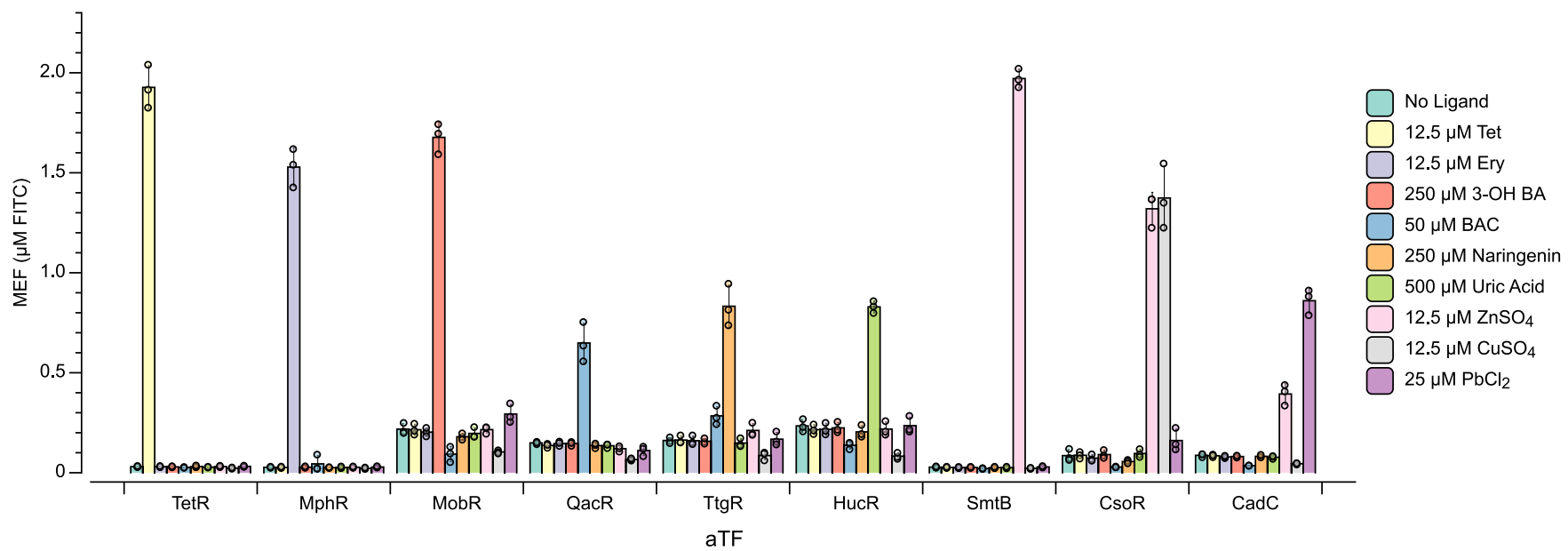

Extended Data Fig. 5 | Orthogonality of ROSALIND reactions. The corresponding bar graph data of the orthogonality matrix shown in Fig. 4a are presented. All data shown for $n=3$ independent biological replicates as points with raw fluorescence values standardized to MEF ( $\mu M$ FITC), and bars representing averages of the replicates. Error bars indicate the average value of 3 independent biological replicates \pm s.d. Tet, Tetracycline; Ery, Erythromycin; 3-OH BA, 3-Hydroxybenzoic Acid; BAC, Benzalkonium chloride. 
a

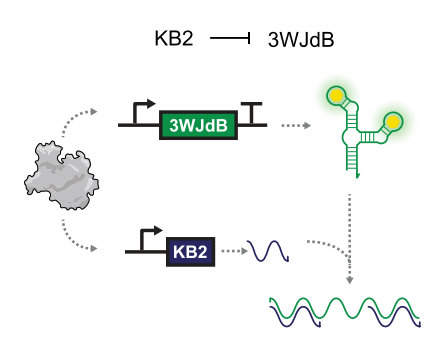

b

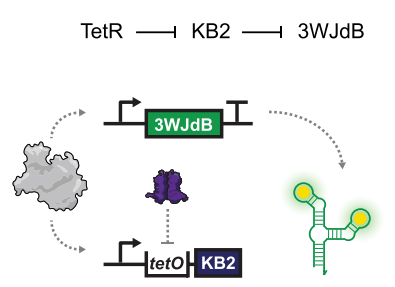

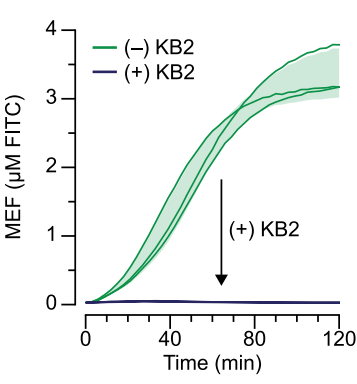

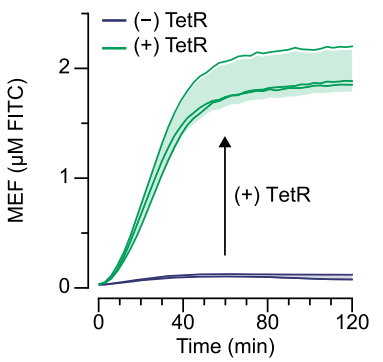

c
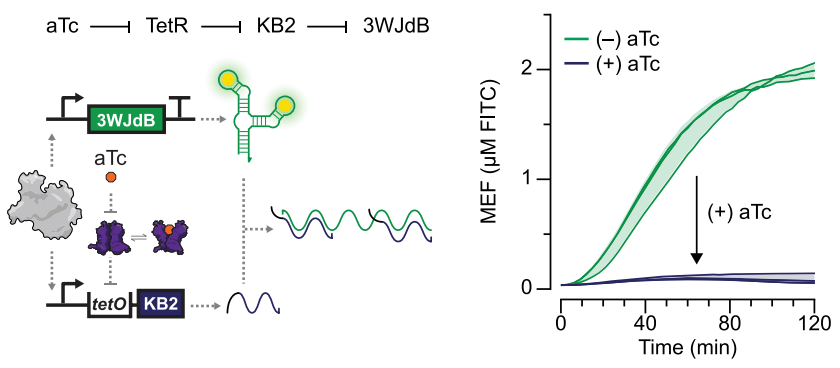

d

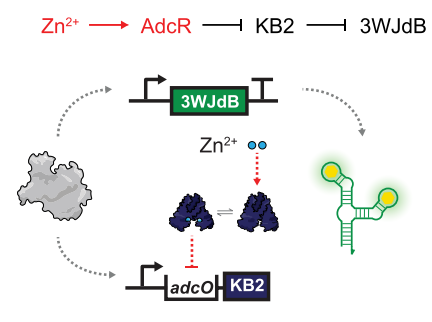

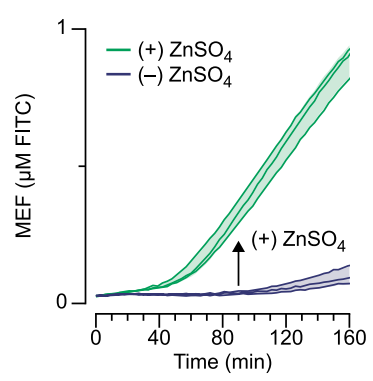

Extended Data Fig. 6 | RNA kleptamers are used to invert transcription factor responses. a, A kleptamer RNA (KB2) antisense to the dye-binding region of the broccoli aptamer can disrupt folding of $3 \mathrm{WJdB}$ and lead to the loss of fluorescence. Addition of a KB2-expressing template in a $4: 1$ ratio with the 3 WJdB template inhibits signal. b, KB2 transcription can be regulated by TetR by placing the tetO site in between the T7 promoter and KB2 coding sequence. In the presence of $1.25 \mu \mathrm{M}$ TetR dimer, the KB2 transcription is fully repressed, and the signal from 3 WJdB is restored. $\mathbf{c}$, Kleptamers can be used to invert the response of transcription factors when KB2 is regulated by TetR ( $1.25 \mu \mathrm{M}$ dimer) and induced by aTc ( $2.5 \mu \mathrm{M}$ shown). $\mathbf{d}$, This scheme was used to create a ROSALIND zinc sensor with the aporepressor AdcR. When bound to $\mathrm{Zn}^{2+}(30 \mu \mathrm{M})$, AdcR (1.5 $\mu \mathrm{M}$ dimer) binds to its cognate operator sequence, adcO, placed upstream of the KB2 coding sequence, preventing KB2 expression and thereby activating fluorescence from 3 WJdB. Arrows inside of the plots represent direction of regulation when indicated species are added. All data shown for $n=3$ independent biological replicates as lines with raw fluorescence values standardized to MEF ( $\mu$ M FITC). Shading indicates the average value of 3 independent biological replicates \pm s.d. 3 WJdB template concentrations used are: $25 \mathrm{nM}$ for $\mathbf{a}-\mathbf{c}$ and $7.5 \mathrm{nM}$ for $\mathbf{d}$. KB2 template concentrations used are: $100 \mathrm{nM}$ for $\mathbf{a}$, and $150 \mathrm{nM}$ for $\mathbf{b}$-d. 


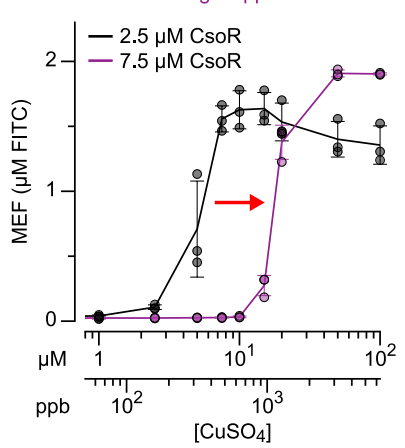

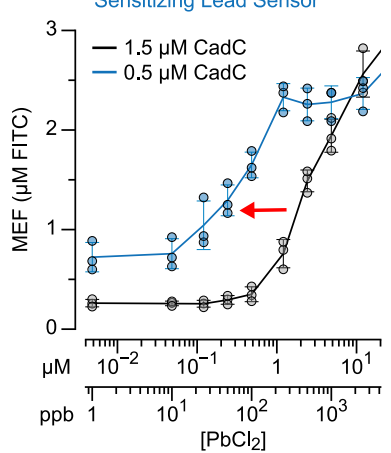

Extended Data Fig. 7 | ROSALIND sensitivity can be tuned by changing the concentration of transcription factor. a, Increasing the concentration of CsoR desensitizes the copper sensor (dose response shift to higher concentrations), while $\mathbf{b}$, decreasing the concentrations of CadC sensitizes the lead sensor (dose response shift to lower concentrations). The red arrows in the plots indicate the direction of dose response shift. All data shown for $\mathrm{n}=3$ independent biological replicates as points with raw fluorescence values standardized to MEF ( $\mu$ M FITC). Error bars indicate the average value of 3 independent biological replicates \pm s.d. 3 WJdB DNA template concentrations used are: $25 \mathrm{nM}$ for $\mathbf{a}$ and $1.5 \mu \mathrm{M}$ CadC condition in $\mathbf{b}$, and $10 \mathrm{nM}$ for $0.5 \mu \mathrm{M}$ CadC condition in $\mathbf{b}$. 


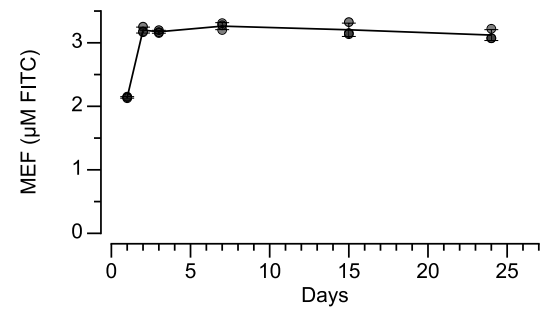

b

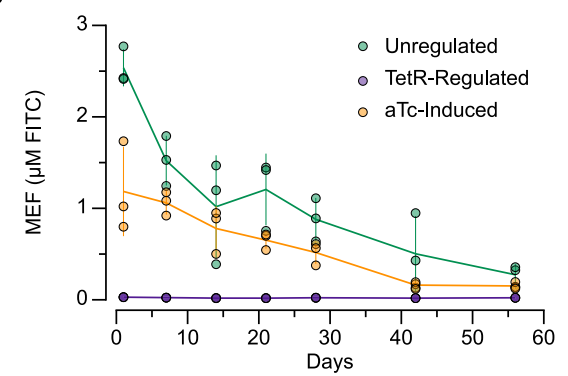

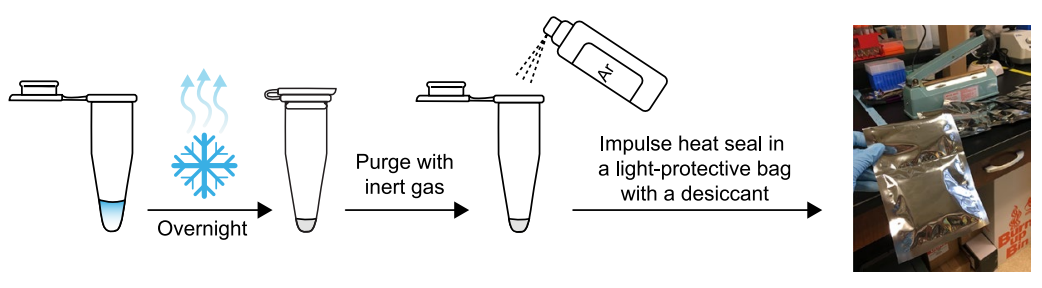

d

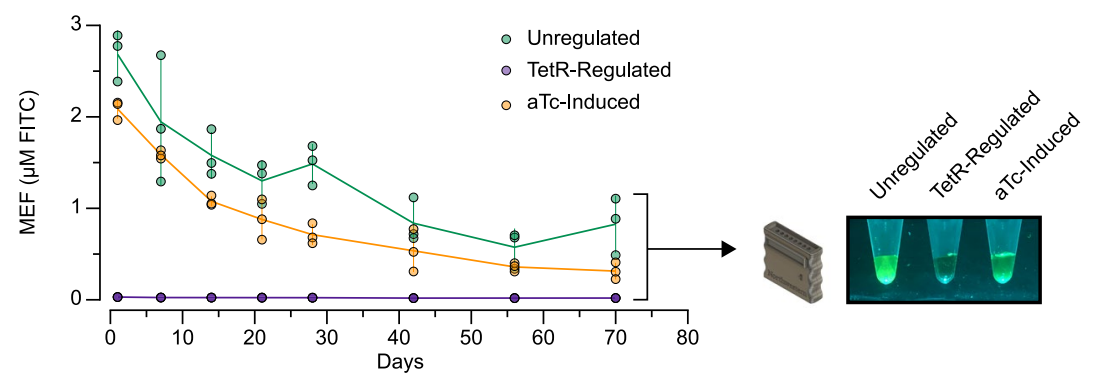

Extended Data Fig. 8 | Stability of ROSALIND. a, The fluorescence signal generated from unregulated ROSALIND reactions are stable over weeks. The increase in fluorescence from Day 1 is likely due to a concentration increase caused by evaporation when the plate was taken out of the incubator after the first measurements. b. The shelf-stability of freeze-dried ROSALIND reactions (unregulated, TetR-regulated and aTc-induced) decay over the course of a month without proper packaging. c, Packaging of freeze-dried ROSALIND: 1) reactions are lyophilized overnight, 2) the overnight lyophilized reactions are purged with inert gas such as argon and 3) the reactions are placed into a light-protective bag with a desiccant and immediately impulse heat sealed (Supplementary Video 2).d, When this packaging method is implemented, lyophilized reactions are functional out to 2.5 months. Though we observed signal decay, the signal from rehydrated reactions after 2.5 months is clearly visible. Images are shown for one replicate with other replicate images included in Supplementary Data 1. Unregulated reactions were lyophilized with $25 \mathrm{nM}$ of the 3 WJdB template, and TetR-regulated reactions with additional $1.25 \mu \mathrm{M}$ TetR dimer along with the components of IVT specified in the In vitro transcription reactions method section. Unregulated and TetR-regulated reactions were then rehydrated with laboratory-grade water, and aTc-induced reactions were rehydrated with $10 \mu M$ of aTc. All data shown for $n=3$ independent biological replicates as points with raw fluorescence values standardized to MEF ( $\mu M$ FITC), and center values representing averages of the replicates. Error bars indicate the average value of 3 independent biological replicates \pm s.d. The original, uncropped images shown in $\mathbf{c}$ and $\mathbf{d}$ can be found in Supplementary Data 1. 
a

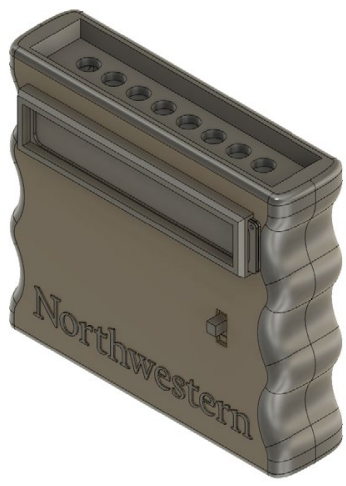

e b

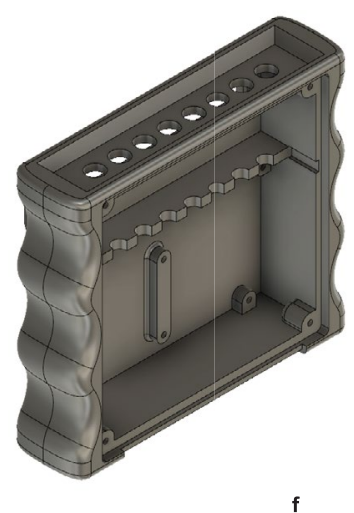

c

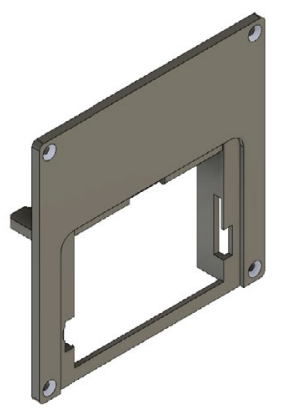

d

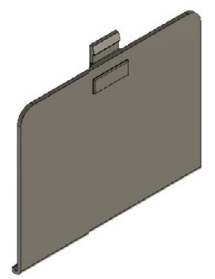

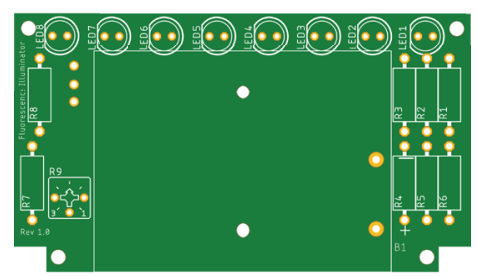

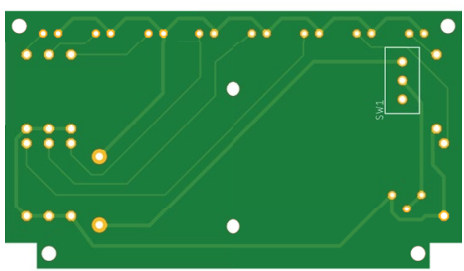

Extended Data Fig. 9 | Handheld fluorescence illuminator design. a, A low-cost handheld fluorescence illuminator enables point-of-use functionality for ROSALIND. 3D printed components include $\mathbf{a}, \mathbf{b}$, front case, $\mathbf{c}$, back case and $\mathbf{d}$, battery cover. A printed circuit board (e, front view facing back case, $\mathbf{f}$, rear view facing front case) mounts in the front case and connects LEDs, resistors, a trimmer potentiometer, a power switch and a battery holder. Full CAD files, 3D print files (.STL), PCB design files and assembly instructions are provided in Supplementary Data 4. The estimated cost of building one device is $\$ 8.69$ USD. 
a

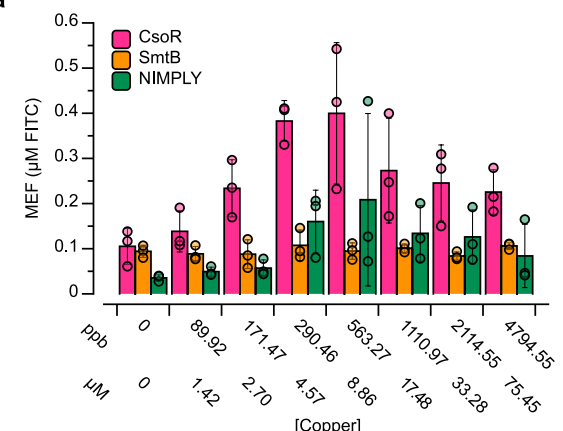

b

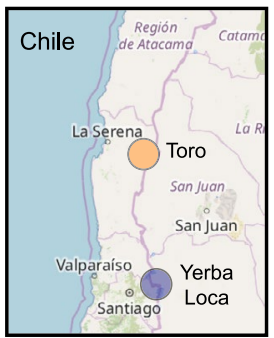

c
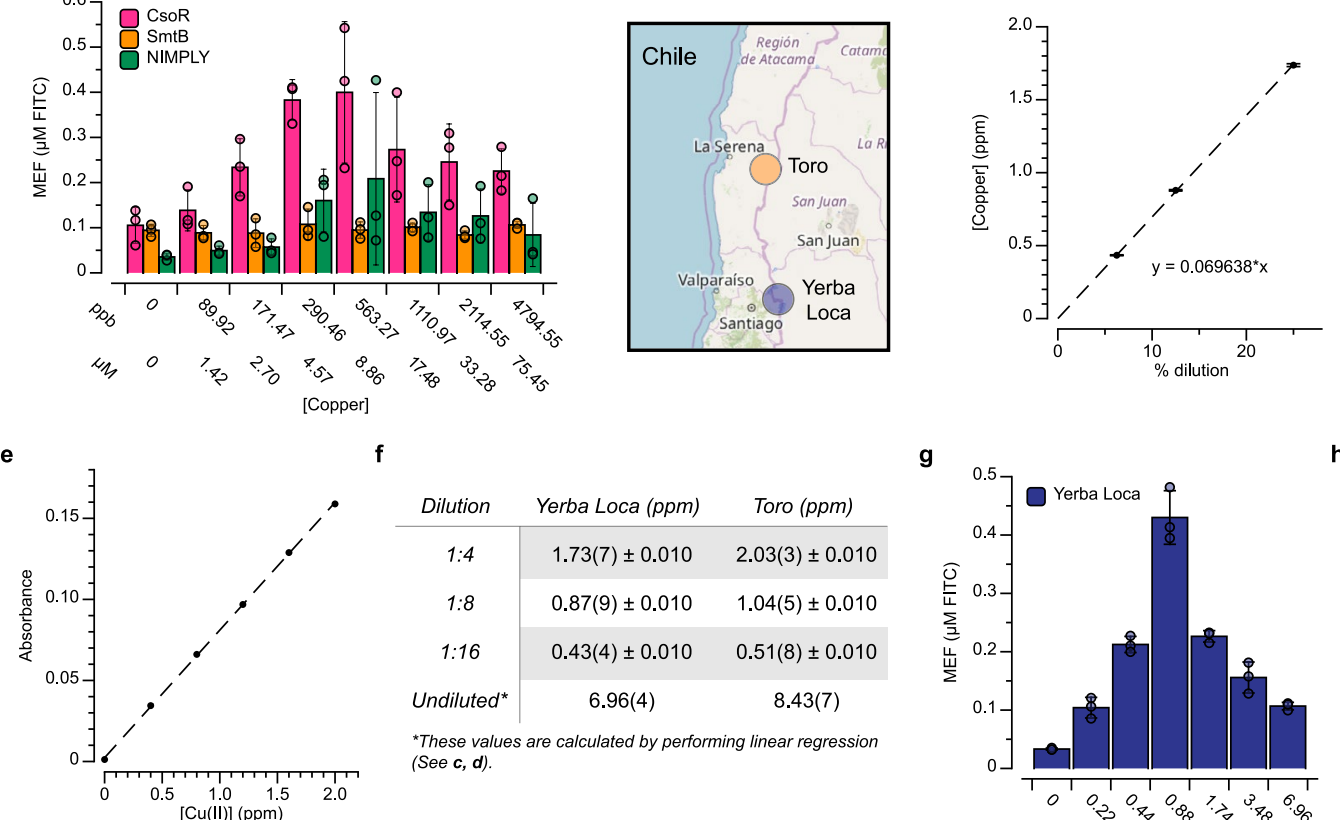

\begin{tabular}{c|cc} 
Dilution & Yerba Loca $(p p m)$ & Toro $(p p m)$ \\
\hline $1: 4$ & $1.73(7) \pm 0.010$ & $2.03(3) \pm 0.010$ \\
$1: 8$ & $0.87(9) \pm 0.010$ & $1.04(5) \pm 0.010$ \\
$1: 16$ & $0.43(4) \pm 0.010$ & $0.51(8) \pm 0.010$ \\
Undiluted & $6.96(4)$ & $8.43(7)$
\end{tabular}

g

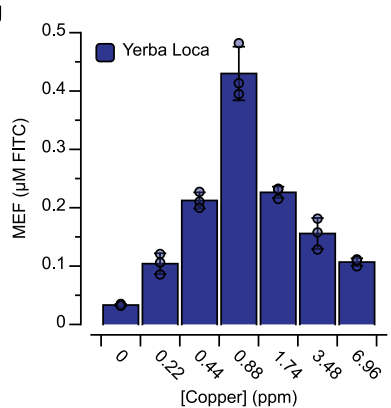

d

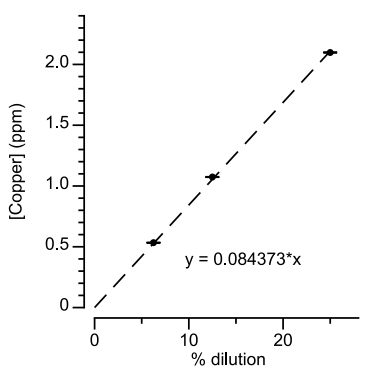

h

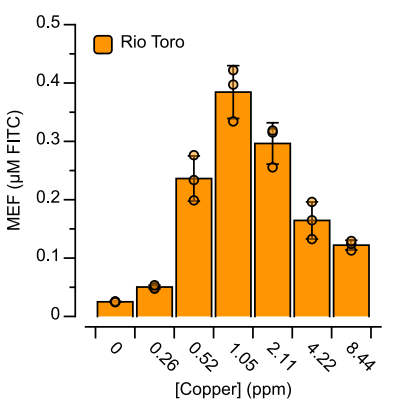

Extended Data Fig. 10 | ROSALIND reactions can be freeze-dried and rehydrated with an environmental surface water source. a, The ROSALIND reactions shown in Fig. 6 were characterized for response to $\mathrm{CuSO}_{4}$ as in Supplementary Fig. 6, but using water from Lake Michigan (collected in Evanston, IL). We observed lower signals from these spiked environmental samples than with the laboratory-grade water samples due to uncharacterized matrix effects. b, To test ROSALIND functionality on real-world environmental samples, surface freshwater samples were obtained from two streams known to contain elevated levels of copper: (1) the Yerba Loca Creek, upstream from Santiago, in the Maipo watershed of Central Chile, and (2) the Toro River, upstream from La Serena, in the Elqui watershed of Northern Chile. c, d, Copper concentrations of the samples from the Yerba Loca Creek (c) and the Toro River (d) were determined using Flame Atomic Absorption Spectroscopy (FAAS) and found to be in the range of 6.9-8.5 ppm. FAAS was calibrated with a $\mathrm{Cu}\left(\mathrm{NO}_{3}\right)_{2}$ standard solution as shown in e. Serial dilution was performed on each sample to create three separate diluted samples that are in the operating ppm range of FAAS, and their ppm values were measured and reported in Table $\mathbf{f}$ as the average value of 3 independent replicates \pm s.d. Using these measurements, linear regression on the averages of each dilution was performed to calculate the ppm value of the undiluted sample. $\mathbf{g}$, $\mathbf{h}$, In order to generate a series of tests that cover our detectable copper range, we serially diluted each sample with laboratory-grade, metal-free water (undiluted, 1:2, 1:4, 1:8, 1:16 and 1:32 dilutions) and measured their concentrations by FAAS before using this series to rehydrate freeze-dried copper sensors. The copper concentrations indicated were taken from c, d, or calculated from the extrapolated ppm value of the undiluted sample in Table f. When rehydrated with the 1:8 dilution, we observed clear visible signals, corresponding to copper concentrations of 0.88 ppm and 1.05 ppm from the Yerba Loca Creek and Toro River samples, respectively. Four-hour end-point data are shown in $\mathbf{a}, \mathbf{g}, \mathbf{h}$ for $\mathbf{n}=3$ independent biological replicates as points with raw fluorescence values standardized to MEF ( $\mu \mathrm{M}$ FITC), and bars representing averages of the replicates. Error bars indicate the average value of 3 independent biological replicates \pm s.d. Geographical data in b (c) OpenStreetMap contributors ${ }^{46}$. 


\section{Reporting Summary}

Nature Research wishes to improve the reproducibility of the work that we publish. This form provides structure for consistency and transparency in reporting. For further information on Nature Research policies, see Authors \& Referees and the Editorial Policy Checklist.

\section{Statistics}

For all statistical analyses, confirm that the following items are present in the figure legend, table legend, main text, or Methods section. n/a Confirmed

$\bigotimes$ The exact sample size $(n)$ for each experimental group/condition, given as a discrete number and unit of measurement

\ A statement on whether measurements were taken from distinct samples or whether the same sample was measured repeatedly

$\triangle$ The statistical test(s) used AND whether they are one- or two-sided

Only common tests should be described solely by name; describe more complex techniques in the Methods section.

Х $\square$ A description of all covariates tested

Х $\square$ A description of any assumptions or corrections, such as tests of normality and adjustment for multiple comparisons

$\triangle$ A full description of the statistical parameters including central tendency (e.g. means) or other basic estimates (e.g. regression coefficient)

AND variation (e.g. standard deviation) or associated estimates of uncertainty (e.g. confidence intervals)

$\varnothing$ For null hypothesis testing, the test statistic (e.g. $F, t, r$ ) with confidence intervals, effect sizes, degrees of freedom and $P$ value noted Give $P$ values as exact values whenever suitable.

Х $\square$ For Bayesian analysis, information on the choice of priors and Markov chain Monte Carlo settings

Х $\square$ For hierarchical and complex designs, identification of the appropriate level for tests and full reporting of outcomes

Х $\square$ Estimates of effect sizes (e.g. Cohen's d, Pearson's $r$ ), indicating how they were calculated

Our web collection on statistics for biologists contains articles on many of the points above.

\section{Software and code}

\section{Policy information about availability of computer code}

$\begin{array}{ll}\text { Data collection } & \begin{array}{l}\text { Biotek Gen5 v2.04 (microplate reader software) } \\ \text { Syngistix (PerkinElmer PinAAcle 500 Flame Atomic Absorption Spectroscopy software) }\end{array}\end{array}$

Data analysis Microsoft Excel for Mac v16

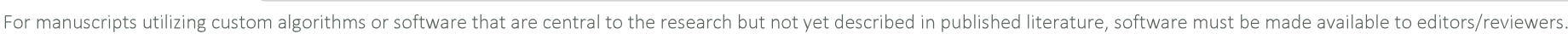
We strongly encourage code deposition in a community repository (e.g. GitHub). See the Nature Research guidelines for submitting code \& software for further information.

\section{Data}

Policy information about availability of data

All manuscripts must include a data availability statement. This statement should provide the following information, where applicable:

- Accession codes, unique identifiers, or web links for publicly available datasets

- A list of figures that have associated raw data

- A description of any restrictions on data availability

\section{All data are provided in Supplementary Data File 3 and will be deposited in Northwestern University's open-access arch database.}

\section{Field-specific reporting}

Please select the one below that is the best fit for your research. If you are not sure, read the appropriate sections before making your selection. 


\section{Life sciences study design}

All studies must disclose on these points even when the disclosure is negative.

Sample size No sample size calculation was used. Sample size of $n=3$ was chosen for quantitative fluorescence measurements based on similar cell-free / in vitro studies. All individual data points are shown in figures, and total sample size spanning all independent experiments is reported in the figure captions.

Data exclusions No data on presented systems were excluded from the analyses. When field testing we did test another preliminary circuit idea we did not discuss in the manuscript. Photographs of these tests are included and annotated in Supplementary Data File 1 (because they were taken alongside the other included circuits), but not discussed in the manuscript.

Replication All experiments were performed three times (independent biological triplicates) by at least one co-author. All attempts at replication were successful.

Randomization While randomization is not relevant to this study since our biochemical reactions are handled uniformly, the same data analysis procedure was applied to all samples of the same type.

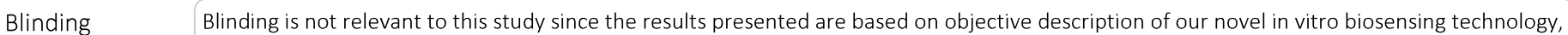
and are therefore not subject to human biases.

\section{Reporting for specific materials, systems and methods}

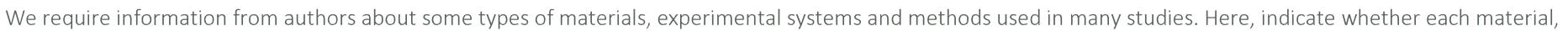

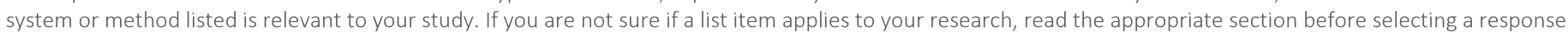

\begin{tabular}{|c|c|}
\hline $\mathrm{n} / \mathrm{a}$ & Involved in the study \\
\hline Х & Antibodies \\
\hline Х & Eukaryotic cell lines \\
\hline Х & Palaeontology \\
\hline Х & Animals and other organisms \\
\hline Х & Human research participants \\
\hline Х & $\square$ Clinical data \\
\hline
\end{tabular}

\begin{tabular}{l|l} 
Methods \\
\hline n/a & Involved in the study \\
$\square$ & $\square$ ChIP-seq \\
$\square$ & $\square$ Flow cytometry \\
$\square$ & $\square$ MRI-based neuroimaging
\end{tabular}

